Pacific Journal of Mathematics

ITERATED AVERAGING FOR PERIODIC SYSTEMS WITH 


\title{
ITERATED AVERAGING FOR PERIODIC SYSTEMS WITH HIDDEN MULTI-SCALE SLOW TIMES
}

\author{
StePhen C. PERSEK
}

\begin{abstract}
General asymptotic methods on various time scales are developed for periodic systems of ordinary differential equations in order to treat global motion in multi-oscillatory systems. Moreover, we show that bifurcations of an attractive and essentially nonperiodic nature can arise in systems that also possess several (often unstable) Hopf bifurcations. Such attractor bifurcations frequently dominate the long term system behavior. In addition, the methods here can be used to determine the flow on a center manifold in cases where center manifold theory indicates an instability at the origin of that manifold and little else about the flow. Finally, various examples of mixed scale motion are treated.
\end{abstract}

1. Introduction. A number of methods have been developed for assessing the behavior of systems of ordinary differential equations. Hopf bifurcation theory as illustrated in J. Marsden and M. McCracken [7] and in A. Poore [13] is a well-known example. And additional results in the treatment of bifurcations have been obtained by $\mathrm{K}$. Landman and S. Rosenblatt [5] and by W. Langford [6]. However, none of the foregoing works comes to grips with bifurcations that either possess an exceptionally large least period or else possess no period at all. This limitation becomes particularly significant for systems possessing two or more characteristic oscillatory frequencies. For instance, considering Example 1 in $\$ 2$ of this paper, Hopf bifurcation theory (see [7, p. 96]) shows the existence of two different periodic bifurcations, the first having a period approximately equal to $2 \pi$ and the second a period approximately equal to $2 \pi / \alpha$ (where 1 and $\alpha$ are the angular frequencies of the system). However, using $\mathrm{S}$. Persek [11], both bifurcations can be shown to be unstable, and thus neither of them characterizes the long term motion of the system. Therefore, in Example 1, we arrange to locate still another bifurcation solution, and as is typical in such cases, this solution is either nonperiodic or, if periodic, has no period smaller in magnitude than order $1 / \varepsilon^{3}$ (where $\varepsilon>0$ is arbitrary and small). Now the fact that no period of this solution can be as small as $2 \pi$ or $2 \pi / \alpha$ rules out the posibility of the solution being a Hopf bifurcation (or even of its being discoverable by that approach). Nevertheless, this solution is a perfectly well-behaved bifurcation which, because of its general characteristics as an attractor, describes the long term motion of the system. In fact, the system will drift away from the 
Hopf bifurcations into the configuration specified by this solution. And finally, using S. Persek [12], this configuration can be shown to be asymptotically stable in an orbital sense. Consequently, the methods developed in this paper will allow us to locate well-behaved (essentially nonperiodic) bifurcations that characterize long term system behavior and frequently coexist with sets of (unstable and therefore not particularly interesting) Hopf bifurcations.

Now another approach often employed in treating systems of ordinary differential equations (whether in a Banach space or a finite-dimensional setting) is center manifold theory, which reduces system behavior to a question of the flow on the center manifold itself, as may be seen in $\mathbf{J}$. Carr [3, pp. 4-5 and pp. 118-120]. If the equation of flow on the center manifold is stable (unstable) at the origin, the original system is also stable (unstable) at this point. However, when the equation of flow is unstable, these results provide little else of a concrete nature as to the ultimate system behavior on the center manifold itself. So at this point, one can turn to Hopf bifurcation theory (whose limitations have already been made clear) or else to Liapunov's direct method (as given by $\mathrm{T}$. Yoshizawa [16], or by N. Rouche, P. Habets, and M. Laloy [14]). The latter method requires a search for an appropriate Liapunov function to characterize the flow, but here it is the flow away from the origin that must be characterized, since, supposedly, center manifold theory has already been used to show the instability of the origin itself. Hence the search may prove quite elusive. So as an alternative, the methods of this paper can be employed to give a detailed description of the flow on the center manifold when Hopf bifurcation theory and Liapunov's methods yield unsatisfactory results - for example, typically in cases where two or more complex conjugate pairs of eigenvalues of the flow equation drift simultaneously across the imaginary axis into the right-hand plane, creating either oscillatory bifurcations or self-excited oscillations of an essentially nonperiodic nature.

Now related to this, N. Bogoliubov and Yu. Mitropolskii [2] developed averaging methods to approximate solutions to periodic systems, with further results by M. Balachandra and P. Sethna [1] and D. Gilsinn [4]. These methods are particularly suited for systems that have an essential nonautonomous structure. Nevertheless, such averaging techniques frequently provide only trivial results, because the lowest order time averages of many systems are zero. In such cases, we can usually obtain far more substantial results.

This paper is a generalization of the method of iterated averaging, introduced by S. Persek and F. Hoppensteadt [8] and extended by S. 
Persek [9] and J. Sanders [15]. The generalization applies to a wide variety of systems, as will be shown in $\$ 2$, and is especially useful for those systems for which Hopf bifurcation theory, center manifold theory, Liapunov's methods, and conventional averaging do not provide particularly suitable or complete results. Finally, iterated averaging as developed here forms the basis of the full and the conditional stability results given in Persek [10], [11], and [12].

We focus on any problem which can be reduced to a system of ordinary differential equations in the form:

$$
\begin{aligned}
\frac{d w_{i}}{d t} & =\varepsilon E_{i}(w, z, t, \varepsilon), \quad 1 \leq i \leq l^{*}, \\
\frac{d z}{d t} & =A z+\varepsilon H(w, z, t, \varepsilon),
\end{aligned}
$$

where $w=\left(w_{1}, w_{2}, \ldots, w_{l^{*}}\right)$ in $R^{l^{*}}$ and $z$ in $R^{m}$ are finite-dimensional column vectors, and where $H$ and the $E_{i}$ 's have a common period $P$ in $t$. Each characteristic root of the constant square matrix $A$ has a negative real part.

With $t=t_{0}(\varepsilon)$ some initial point, and with

$$
z^{(0)}(t)=e^{A\left(t-t_{0}\right)} z\left(t_{0}\right)
$$

integrating equations (la), (1b) leads to:

$$
\begin{gathered}
w_{i}(t)=\rho_{i}+\varepsilon \int_{s}^{t} E_{i}(w(\tau), z(\tau), \tau, \varepsilon) d \tau, \quad 1 \leq i \leq l^{*}, \\
z(t)=z^{(0)}(t)+\varepsilon \int_{t_{0}}^{t} e^{A(t-\tau)} H(w(\tau), z(\tau), \tau, \varepsilon) d \tau,
\end{gathered}
$$

where $s$ is any point with $t_{0} \leq s \leq t$, and $\rho_{i}=w_{l}(s)$. Let $\rho=$ $\left(\rho_{1}, \rho_{2}, \ldots, \rho_{l^{*}}\right)$. By Taylor's theorem, we expand each $E_{l}$ and $H$ about $(w, z, t, \varepsilon)=(\rho, 0, t, 0)$. Then repeated iteration of (2) and (3) allows us to write

$$
\varepsilon E_{l}(w(t), z(t), t, \varepsilon)=\sum_{j=1}^{n_{l}} \varepsilon^{j} E_{i, j}\left(\rho, t, s, t_{0}\right)+\varepsilon R_{i, n_{l}}
$$

for $1 \leq i \leq l^{*}$, where the $E_{i, j}$ depend on the initial points $s$ and $t_{0}$ but not on $\varepsilon$ (other than through $t_{0}$ and $s$ ) nor on $z^{(0)}(t)$, and where $\varepsilon R_{i, n_{i}}$ is the remainder. The dependence of each $E_{i, j}$ on $(\rho, 0, t, 0)$ and on the initial points $s$ and $t_{0}$ has been indicated by writing $E_{i, j}=E_{i, j}\left(\rho, t, s, t_{0}\right)$.

If the various $n_{i}$ are chosen properly, then for a given $i$ and all $j \leq n_{i}$, each $\left.E_{i, j}\left(\rho, t, s, t_{0}\right)\right|_{t_{0}=-\infty}$ is periodic in $t$ with period $P$, and for $j<n_{i}$, the average of $\left.E_{l, j}\left(\rho, t, s, t_{0}\right)\right|_{t_{0}=-\infty}$ with respect to $t$ is identically zero. 
We then define $\bar{E}_{i, n_{i}}$ as the average of $\left.E_{l, n_{t}}\left(\rho, t, s, t_{0}\right)\right|_{t_{0}=-\infty}$ with respect to $t$. Having obtained the averages $\bar{E}_{i, n_{t}}(\rho)$ for $1 \leq i \leq l^{*}$, we now repeal the assumption made earlier that the vector $\rho$ was the initial value of $w$ at $t=s$, and we instead formulate the "iterated-average" system:

$$
\begin{aligned}
\frac{d \rho_{l}}{d t} & =\varepsilon^{n_{i}} \bar{E}_{i, n_{i}}(\rho), \quad 1 \leq i \leq l^{*}, \\
\frac{d \zeta}{d t} & =A \zeta,
\end{aligned}
$$

where the integers $n_{t}$ may (or may not) differ in value from one another and where $\zeta$ lies in $R^{m}$. (Note that the $\bar{E}_{l, n_{1}}(\rho)$ are usually independent of $s$.)

Now in problems where angular variables appear, certain components of $\rho$ may represent angular shifts, say $\rho_{l+1}, \rho_{l+2}, \ldots, \rho_{1^{*}}$. And so it frequently happens that for $i \leq l$, the $\bar{E}_{l, n_{t}}(\rho)$ are independent of these $\rho$-components. If, further, it is true that all the $E_{l}(w, z, t, \varepsilon)$ and $H(w, z, t, \varepsilon)$ are uniformly bounded in the corresponding $w$-components $w_{l+1}, w_{l+2}, \ldots, w_{l^{*}}$, we then define the vector $\rho_{+}$in $R^{l}$ by $\rho_{+}=$ $\left(\rho_{1}, \rho_{2}, \ldots, \rho_{l}\right)$, and consistent with this, rewrite the averages in the form $\bar{E}_{i, n_{i}}=\bar{E}_{i, n_{t}}\left(\rho_{+}\right)$for $1 \leq i \leq l$. The iterated-average system (at least the portion of it we are concerned with) then becomes:

$$
\begin{aligned}
\frac{d \rho_{i}}{d t} & =\varepsilon^{n} \bar{E}_{i, n_{i}}\left(\rho_{+}\right), \quad 1 \leq i \leq l, \\
\frac{d \zeta}{d t} & =A \zeta
\end{aligned}
$$

and system (5a) and (5b) is the system our results have been developed for. (We could recover the full $\rho$-system by simply letting $l=l^{*}$ so that $\left.\rho_{+} \equiv \rho.\right)$

With $w_{+}=\left(w_{1}, w_{2}, \ldots, w_{l}\right)$, the main result of this paper is that a solution of $\left(\rho_{+}, \zeta\right)$ to system $(5 \mathrm{a}),(5 \mathrm{~b})$ approximates the $\left(w_{+}, z\right)$ components of a solution to system (1a), (1b) to order $\varepsilon$ for any small $\varepsilon \geq 0$, provided the values of $\left(\rho_{+}, \zeta\right)$ and $\left(w_{+}, z\right)$ chosen at $t=t_{0}$ are equal. The approximation is uniform on the interval $t_{0} \leq t \leq t_{0}+O\left(\varepsilon^{-\max n_{2}}\right)$, if the $\left(\rho_{+}, \zeta\right)$ solution on this interval remains in a fixed bounded region for small $\varepsilon>0$. Finally, the approximation is uniform on $t_{0} \leq t<\infty$, if the $\left(\rho_{+}, \zeta\right)$ system is exponentially asymptotically stable and if $\left.\left(w_{+}, z\right)\right|_{t=t_{0}}$ lies in the domain of stability of the $\left(\rho_{+}, \zeta\right)$ system.

The complete results are given in $\$ 4$, and the precise statement of the conditions necessary for the results to apply is found earlier in \$3. Moreover, as will be seen in $\$ 2$, application can be made to locating 
well-behaved periodic and nonperiodic bifurcations of nonautonomous and autonomous systems. Lastly, we wish to point out that the reader may find it easier to calculate the averages $\bar{E}_{i, n_{l}}$ from the equations

$$
\begin{aligned}
& r_{i}(t)=\rho_{l}+\varepsilon \int_{s}^{t} E_{l}(r(\tau), q(\tau), \tau, \varepsilon) d \tau, \quad 1 \leq i \leq l^{*}, \\
& q(t)=\varepsilon \int_{-\infty}^{t} e^{A(t-\tau)} H(r(\tau), q(\tau), \tau, \varepsilon) d \tau,
\end{aligned}
$$

with $r=\left(r_{1}, r_{2}, \ldots, r_{l^{*}}\right)$ in $R^{l^{*}}$ and with $q$ in $R^{m}$, than from equations (2) and (3).

2. Applications. Without resorting to center manifold theory, we first examine a well-behaved chaotic bifurcation (coexisting with two unstable Hopf bifurcations, as seen from [7, p. 96] and [11]).

EXAMPLE 1. With $\alpha$ and $\varepsilon$ positive scalars ( $\alpha$ rational) and with the column vector $x=\left(x_{1}, x_{2}, x_{3}, x_{4}, x_{5}\right)$, consider the system

$$
\frac{d x}{d t}=\left(\begin{array}{ccccc}
\varepsilon^{3} & -\alpha & 0 & 0 & 0 \\
\alpha & \varepsilon^{3} & 0 & 0 & 0 \\
0 & 0 & \varepsilon^{3} & -1 & 0 \\
0 & 0 & 1 & \varepsilon^{3} & 0 \\
0 & 0 & 0 & 0 & -1
\end{array}\right) x+\left(\begin{array}{c}
x_{2} x_{5}^{2} \\
0 \\
0 \\
-x_{4} x_{5}^{2} \\
H^{*}\left(x_{1}, x_{2}, x_{3}, x_{4}\right)
\end{array}\right)
$$

where $H^{*}$ is linear in its variables. Employing the scaled transformations

$$
\begin{aligned}
& x_{1}=\varepsilon^{3 / 2} w_{1} \cos \left(\alpha t+w_{3}\right), \\
& x_{2}=\varepsilon^{3 / 2} w_{1} \sin \left(\alpha t+w_{3}\right), \\
& x_{3}=\varepsilon^{3 / 2} w_{2} \cos \left(t+w_{4}\right), \\
& x_{4}=\varepsilon^{3 / 2} w_{2} \sin \left(t+w_{4}\right), \\
& x_{5}=\varepsilon^{1 / 2} z,
\end{aligned}
$$

we have:

$$
\begin{aligned}
& \frac{d w_{1}}{d t}=\varepsilon^{3} w_{1}+\varepsilon w_{1} z^{2} \sin \left(\alpha t+w_{3}\right) \cos \left(\alpha t+w_{3}\right), \\
& \frac{d w_{2}}{d t}=\varepsilon^{3} w_{2}-\varepsilon w_{2} z^{2} \sin ^{2}\left(t+w_{4}\right), \\
& \frac{d w_{3}}{d t}=-\varepsilon z^{2} \sin ^{2}\left(\alpha t+w_{3}\right), \\
& \frac{d w_{4}}{d t}=-\varepsilon z^{2} \sin \left(t+w_{4}\right) \cos \left(t+w_{4}\right), \\
& \frac{d z}{d t}=-z+\varepsilon H\left(w_{1}, w_{2}, w_{3}, w_{4}, t\right),
\end{aligned}
$$


where $H=\varepsilon^{-3 / 2} H^{*}$. With $w=\left(w_{1}, w_{2}, w_{3}, w_{4}\right)$, let the right-hand side of the equation for each $w_{l}$ be $\varepsilon E_{i}(w, z, t, \varepsilon)$ and let $\rho=\left(\rho_{1}, \rho_{2}, \rho_{3}, \rho_{4}\right)$. The reader will then find that iterating equations (2) and (3) as outlined in $\S 1$ and then averaging to obtain the $\bar{E}_{i, n_{l}}(\rho)$ reduce (in this example) to evaluating

$$
\begin{aligned}
\bar{E}_{i, 3}(\rho)= & \left.\frac{1}{2 \mu \pi} \int_{t_{0}}^{2 \mu \pi+t_{0}} \frac{1}{2 !}\left(\frac{\partial^{2} E_{l}}{\partial \varepsilon^{2}}\right)(\rho, 0, \tau, \varepsilon)\right|_{\varepsilon=0} d \tau \\
& +\left.\frac{1}{2 \mu \pi} \int_{t_{0}}^{2 \mu \pi+t_{0}} \frac{1}{2 !}\left(\frac{\partial^{2} E_{i}}{\partial z^{2}}\right)(\rho, z, \tau, 0)\right|_{z=0} \\
& \times\left\{\int_{-\infty}^{\tau} e^{-(\tau-\sigma)} H\left(\rho_{1}, \rho_{2}, \rho_{3}, \rho_{4}, \sigma\right) d \sigma\right\}^{2} d \tau
\end{aligned}
$$

where $\mu$ is the smallest positive integer such that $\mu \alpha$ is an integer. In particular, with $H^{*}\left(x_{1}, x_{2}, x_{3}, x_{4}\right)=\beta_{1} x_{1}+\beta_{2} x_{2}+\beta_{3} x_{3}+\beta_{4} x_{4}$ (the $\beta_{j}$ constant), we obtain the iterated average system:

$$
\begin{aligned}
& \frac{d \rho_{1}}{d t}=\varepsilon^{3}\left\{\rho_{1}-\frac{\left(\alpha \beta_{2}-\beta_{1}\right)\left(\beta_{2}+\alpha \beta_{1}\right)}{4\left(1+\alpha^{2}\right)^{2}} \rho_{1}^{3}\right\}, \\
& \frac{d \rho_{2}}{d t}=\varepsilon^{3}\left\{\rho_{2}-\frac{\beta_{3}^{2}+\beta_{3} \beta_{4}+\beta_{4}^{2}}{8} \rho_{2}^{3}\right\}, \\
& \frac{d \rho_{3}}{d t}=-\frac{\varepsilon^{3}}{8} \rho_{1}^{2}\left\{\frac{3\left(\alpha \beta_{1}+\beta_{2}\right)^{2}+\left(\beta_{1}-\alpha \beta_{2}\right)^{2}}{\left(1+\alpha^{2}\right)^{2}}\right\}, \\
& \frac{d \rho_{4}}{d t}=-\frac{\varepsilon^{3}}{16} \rho_{2}^{2}\left(\beta_{3}^{2}-\beta_{4}^{2}\right), \\
& \frac{d \zeta}{d t}=-\zeta,
\end{aligned}
$$

provided $\alpha$, which is rational, is not zero or one. Then from $\S 1$ or from the Theorem of $\S 4$, it follows that for all small $\varepsilon>0$ and any $t_{0} \geq 0$ :

$$
\sup _{t_{0} \leq t \leq t_{0}+M / \varepsilon^{3}}\left\{\sum_{i=1}^{4}\left|w_{l}(t, \varepsilon)-\rho_{i}(t, \varepsilon)\right|+|z(t, \varepsilon)-\zeta(t, \varepsilon)|\right\} \leq K(M) \varepsilon
$$

where $K(M)$ depends on $M$, but not on $\varepsilon$ or $t_{0}$ or on any initial value of $(w, z)$ selected from a fixed bounded region. 
Now concentrating instead on the $\left(\rho_{1}, \rho_{2}, \zeta\right)$ subsystem, we note that it has an exponentially asymptotically stable rest point at

$$
\left(\rho_{1}, \rho_{2}, \zeta\right)=\left(\frac{2\left(1+\alpha^{2}\right)}{\sqrt{\left(\alpha \beta_{2}-\beta_{1}\right)\left(\beta_{2}+\alpha \beta_{1}\right)}}, \frac{2 \sqrt{2}}{\sqrt{\beta_{3}^{2}+\beta_{3} \beta_{4}+\beta_{4}^{2}}}, 0\right)
$$

provided $\alpha\left(\beta_{2}^{2}-\beta_{1}^{2}\right)+\left(\alpha^{2}-1\right) \beta_{1} \beta_{2}>0$. Consequently, in this case, we have, for all small $\varepsilon>0$,

$$
\begin{aligned}
\sup _{t_{0} \leq t<\infty}\left\{\left|w_{1}(t, \varepsilon)-\rho_{1}(t, \varepsilon)\right|+\mid w_{2}(t, \varepsilon)\right. & -\rho_{2}(t, \varepsilon) \mid \\
& +|z(t, \varepsilon)-\zeta(t, \varepsilon)|\} \leq K^{*} \varepsilon
\end{aligned}
$$

where $K^{*}$ does not depend on $\varepsilon$ or $t_{0}$ or on the initial value of $(w, z)$, provided the initial value of $\left(w_{1}, w_{2}, z\right)$ is not selected from outside of a fixed bounded domain of stability of the $\left(\rho_{1}, \rho_{2}, \zeta\right)$ subsystem.

Because of the foregoing analysis, the original $x$-system has a family of solutions bifurcating from $x=0$ for all small $\varepsilon>0$, whenever $\alpha\left(\beta_{2}^{2}-\beta_{1}^{2}\right)+\left(\alpha^{2}-1\right) \beta_{1} \beta_{2}>0$, and $\beta_{3}^{2}+\beta_{4}^{2}>0$. The $x$-components of the solution bifurcating from $x=0$ for a particular value of $\varepsilon$ satisfy

$$
\begin{aligned}
& x_{1}^{2}+x_{2}^{2}=\frac{4 \varepsilon^{3}\left(1+\alpha^{2}\right)^{2}}{\left(\alpha \beta_{2}-\beta_{1}\right)\left(\beta_{2}+\alpha \beta_{1}\right)}+O\left(\varepsilon^{4}\right), \\
& x_{3}^{2}+x_{4}^{2}=\frac{8 \varepsilon^{3}}{\beta_{3}^{2}+\beta_{3} \beta_{4}+\beta_{4}^{2}}+O\left(\varepsilon^{4}\right),
\end{aligned}
$$

and it can be shown that $x_{5}$ is the sum of two essentially oscillatory functions, the first with the maximum amplitude

$$
\sqrt{\beta_{1}^{2}+\beta_{2}^{2}} \sqrt{x_{1}^{2}+x_{2}^{2}} / \sqrt{1+\alpha^{2}}+O\left(\varepsilon^{5 / 2}\right),
$$

and the second with the maximum amplitude

$$
\sqrt{\beta_{3}^{2}+\beta_{4}^{2}} \sqrt{x_{3}^{2}+x_{4}^{2}} / \sqrt{2}+O\left(\varepsilon^{5 / 2}\right) .
$$

Moreover, aside from a few special cases of no concern to us here, the $\left(\rho_{3}, \rho_{4}\right)$ equations show that the bifurcated solution either is nonperiodic for small $\varepsilon>0$, or its smallest possible period is $O\left(1 / \varepsilon^{3}\right)$. Hence by [7, p. 96], this solution cannot be a Hopf bifurcation. And clearly, any trajectory that approaches sufficiently near the orbit of our bifurcated solution becomes trapped about that orbit. Moreover by [12], this bifurcation is asymptotically stable in an orbital sense. And finally, note that the preceding analysis is fully valid for any rational $\alpha>0$ with $\alpha \neq 1$. 
EXAMPLE 2. (Multiple Frequency Quadratic Bifurcation). Quadratic systems are used, among other things, to describe predator-prey interactions in ecological settings. Here, we treat a well-behaved chaotic bifurcation, coexisting with two Hopf bifurcations - at least one of them obviously unstable (see [7, p. 96] and [11]). Note that our approach would apply just as well to a problem with an even larger number of oscillatory degrees of freedom.

Let $x=\left(x_{1}, x_{2}, x_{3}, x_{4}\right)$ be a column vector. Then with the $\alpha_{k}$ and $\beta_{k}$ constant, consider the system

$$
\frac{d x}{d t}=\left(\begin{array}{cccc}
\varepsilon^{2} & -1 & 0 & 0 \\
1 & \varepsilon^{2} & 0 & 0 \\
0 & 0 & \varepsilon^{2} & -\gamma \\
0 & 0 & \gamma & \varepsilon^{2}
\end{array}\right) x+\left(\begin{array}{c}
\alpha_{1} x_{2}^{2}+\beta_{1} x_{3}^{2} \\
-\alpha_{2} x_{2}^{2} \\
\alpha_{3} x_{4}^{2}+\beta_{2} x_{1} x_{3} \\
-\alpha_{4} x_{4}^{2}
\end{array}\right)
$$

where $\gamma$ is any fixed positive rational. Letting

$$
\begin{aligned}
& x_{1}=\varepsilon w_{1} \cos \left(t+w_{3}\right), \\
& x_{2}=\varepsilon w_{1} \sin \left(t+w_{3}\right), \\
& x_{3}=\varepsilon w_{2} \cos \left(\gamma t+w_{4}\right), \\
& x_{4}=\varepsilon w_{2} \sin \left(\gamma t+w_{4}\right),
\end{aligned}
$$

we obtain:

$$
\begin{aligned}
\frac{d w_{1}}{d t}= & \varepsilon^{2} w_{1}+\varepsilon \alpha_{1} w_{1}^{2} \cos \left(t+w_{3}\right) \sin ^{2}\left(t+w_{3}\right)-\varepsilon \alpha_{2} w_{1}^{2} \sin ^{3}\left(t+w_{3}\right) \\
& +\varepsilon \beta_{1} w_{2}^{2} \cos \left(t+w_{3}\right) \cos ^{2}\left(\gamma t+w_{4}\right) \\
\frac{d w_{2}}{d t}= & \varepsilon^{2} w_{2}+\varepsilon \alpha_{3} w_{2}^{2} \cos \left(\gamma t+w_{4}\right) \sin ^{2}\left(\gamma t+w_{4}\right)-\varepsilon \alpha_{4} w_{2}^{2} \sin ^{3}\left(\gamma t+w_{4}\right) \\
& +\varepsilon \beta_{2} w_{1} w_{2} \cos \left(t+w_{3}\right) \cos ^{2}\left(\gamma t+w_{4}\right) \\
\frac{d w_{3}}{d t}= & -\varepsilon \alpha_{2} w_{1} \cos \left(t+w_{3}\right) \sin ^{2}\left(t+w_{3}\right)-\varepsilon \alpha_{1} w_{1} \sin ^{3}\left(t+w_{3}\right) \\
& -\varepsilon \frac{\beta_{1} w_{2}^{2}}{w_{1}} \sin \left(t+w_{3}\right) \cos ^{2}\left(\gamma t+w_{4}\right) \\
\frac{d w_{4}}{d t}= & -\varepsilon \alpha_{4} w_{2} \cos \left(\gamma t+w_{4}\right) \sin ^{2}\left(\gamma t+w_{4}\right)-\varepsilon \alpha_{3} w_{2} \sin ^{3}\left(\gamma t+w_{4}\right) \\
& -\varepsilon \beta_{2} w_{1} \cos \left(t+w_{3}\right) \sin \left(\gamma t+w_{4}\right) \cos \left(\gamma t+w_{4}\right) .
\end{aligned}
$$

With $w=\left(w_{1}, w_{2}, w_{3}, w_{4}\right)$, let $\varepsilon E_{i}(w, t, \varepsilon)$ be the right-hand side of each $w_{i}$-equation, and write $\rho=\left(\rho_{1}, \rho_{2}, \rho_{3}, \rho_{4}\right)$. Then iterating equations (2) 
and (3) as outlined in $\$ 1$ and averaging to obtain the $\bar{E}_{i, n_{l}}$ reduces in this problem to evaluating

$$
\begin{aligned}
\bar{E}_{i, 2}= & \left.\frac{1}{2 \pi \mu} \int_{t_{0}}^{2 \pi \mu+t_{0}}\left(\frac{\partial E_{i}}{\partial \varepsilon}\right)(\rho, \tau, \varepsilon)\right|_{\varepsilon=0} d \tau \\
& +\left.\frac{1}{2 \pi \mu} \int_{t_{0}}^{2 \pi \mu+t_{0}} \sum_{j=1}^{4}\left\{\left(\frac{\partial E_{i}}{\partial w_{j}}\right)(w, \tau, 0) \int_{t_{0}}^{\tau} E_{j}(w, \sigma, 0) d \sigma\right\}\right|_{w=\rho} d \tau,
\end{aligned}
$$

where $\mu>0$ is the smallest integer such that $\gamma \mu$ is an integer as well. We therefore have the iterated average systems:

$$
\begin{aligned}
& \frac{d \rho_{1}}{d t}=\varepsilon^{2} \rho_{1}\left(1-\frac{1}{4} \alpha_{1} \alpha_{2} \rho_{1}^{2}-\frac{1}{2} \alpha_{2} \beta_{1} \rho_{2}^{2}\right), \\
& \frac{d \rho_{2}}{d t}=\varepsilon^{2} \rho_{2}\left(1+\frac{1}{4} \alpha_{2} \beta_{2} \rho_{1}^{2}-\frac{1}{4 \gamma} \alpha_{3} \alpha_{4} \rho_{2}^{2}\right), \\
& \frac{d \rho_{3}}{d t}=-\frac{\varepsilon^{2}}{12}\left(5 \alpha_{1}^{2}+2 \alpha_{2}^{2}\right) \rho_{1}^{2}-\frac{\varepsilon^{2}}{2} \beta_{1} \rho_{2}^{2}\left(\alpha_{1}+\frac{2 \gamma^{2}-1}{4 \gamma^{2}-1} \beta_{2}\right), \\
& \frac{d \rho_{4}}{d t}=-\frac{\varepsilon^{2}}{12 \gamma}\left(5 \alpha_{3}^{2}+2 \alpha_{4}^{2}\right) \rho_{2}^{2}-\frac{\varepsilon^{2}}{4} \frac{\gamma}{4 \gamma^{2}-1} \beta_{2}\left(\beta_{2} \rho_{1}^{2}+\beta_{1} \rho_{2}^{2}\right),
\end{aligned}
$$

povided the rational value chosen for $\gamma$ is not $\frac{1}{3}, \frac{1}{5}, 1, \frac{1}{2}$, or 2 .

Then from $\$ 1$ or from the Theorem of $\S 4$, it follows that for any $t_{0} \geq 0$ and for all small $\varepsilon>0$

$$
\sup _{t_{0} \leq t \leq t_{0}+M / \varepsilon^{2}}\left\{\sum_{i=1}^{4}\left|w_{i}(t, \varepsilon)-\rho_{i}(t, \varepsilon)\right|\right\} \leq K(M) \varepsilon,
$$

where $K(M)$ depends on the choice of $M$, but not on $t_{0}$ or $\varepsilon$ or on the initial value of $w$ (as long as the initial value choice is confined to some fixed bounded region).

Now concentrating instead on the $\left(\rho_{1}, \rho_{2}\right)$ subsystem, we find an exponentially asymptotically stable rest point located at

$$
\left(\rho_{1}, \rho_{2}\right)=\left(\sqrt{\frac{4 \alpha_{3} \alpha_{4}-8 \gamma \alpha_{2} \beta_{1}}{\alpha_{1} \alpha_{2} \alpha_{3} \alpha_{4}+2 \gamma \alpha_{2}^{2} \beta_{1} \beta_{2}}}, \sqrt{\frac{4 \gamma \alpha_{1} \alpha_{2}+4 \gamma \alpha_{2} \beta_{2}}{\alpha_{1} \alpha_{2} \alpha_{3} \alpha_{4}+2 \gamma \alpha_{2}^{2} \beta_{1} \beta_{2}}}\right)
$$

provided that both square roots are real and positive. Consequently, in this case, we have for all small $\varepsilon>0$,

$$
\sup _{t_{0} \leq t<\infty}\left\{\left|w_{1}(t, \varepsilon)-\rho_{1}(t, \varepsilon)\right|+\left|w_{2}(t, \varepsilon)-\rho_{2}(t, \varepsilon)\right|\right\} \leq K^{*} \varepsilon,
$$


where $K^{*}$ is independent of $\varepsilon$ and $t_{0}$ and does not depend on which initial value of $\left(w_{1}, w_{2}\right)$ is chosen (as long as the choice is confined to a fixed domain of stability of the $\left(\rho_{1}, \rho_{2}\right)$ subsystem).

By the preceding analysis then, the quadratic system for $x$ has a solution bifurcating from $x=0$ for all small $\varepsilon>0$, as long as a rest point $\left(a_{1}, a_{2}\right)$ exists for the $\left(\rho_{1}, \rho_{2}\right)$ subsystem and satisfies $a_{1}>0, a_{2}>0$. The components of that solution satisfy:

$$
\begin{aligned}
& x_{1}^{2}+x_{2}^{2}=\frac{4 \alpha_{3} \alpha_{4}-8 \gamma \alpha_{2} \beta_{1}}{\alpha_{1} \alpha_{2} \alpha_{3} \alpha_{4}+2 \gamma \alpha_{2}^{2} \beta_{1} \beta_{2}} \varepsilon^{2}+O\left(\varepsilon^{3}\right), \\
& x_{3}^{2}+x_{4}^{2}=\frac{4 \gamma \alpha_{1} \alpha_{2}+4 \gamma \alpha_{2} \beta_{2}}{\alpha_{1} \alpha_{2} \alpha_{3} \alpha_{4}+2 \gamma \alpha_{2}^{2} \beta_{1} \beta_{2}} \varepsilon^{2}+O\left(\varepsilon^{3}\right)
\end{aligned}
$$

Moreover, from the $\left(\rho_{3}, \rho_{4}\right)$ equations, we see that the solution bifurcating from $x=0$ is either nonperiodic or has a smallest possible period of $O\left(1 / \varepsilon^{2}\right)$ for small $\varepsilon>0$ (aside from a few special cases which don't interest us). Hence by [7, p. 96], this solution cannot be a Hopf bifurcation. And clearly, any trajectory that approaches sufficiently near the orbit of our bifurcated solution becomes trapped about that orbit. Moreover by [12], this bifurcation is asymptotically stable in an orbital sense. And finally, note that the preceding analysis is fully valid for any rational $\gamma>0$, provided $\gamma \neq \frac{1}{5}, \frac{1}{3}, \frac{1}{2}, 1$, or 2 . These latter $\gamma$-values can also be treated, but we omit this.

EXAMPLE 3. (Bifurcation in a Nonautonomous System.) With $\alpha_{1}, \alpha_{2}, \alpha_{3}$, $\alpha_{4}$ constants, consider the system:

$$
\begin{aligned}
& \frac{d x_{1}}{d t}=\varepsilon^{2} x_{1}+x_{2}^{2} \sin t+\left(\alpha_{1} x_{1}^{2}+\alpha_{2} x_{1} x_{2}+\alpha_{3} x_{2}^{2}\right) \cos t \\
& \frac{d x_{2}}{d t}=\varepsilon^{2} x_{2}+\alpha_{4} x_{1} x_{2} \cos t
\end{aligned}
$$

By the substitutions $x_{1}=\varepsilon w_{1}, x_{2}=\varepsilon w_{2}$, we have:

$$
\begin{aligned}
& \frac{d w_{1}}{d t}=\varepsilon^{2} w_{1}+\varepsilon w_{2}^{2} \sin t+\varepsilon\left(\alpha_{1} w_{1}^{2}+\alpha_{2} w_{1} w_{2}+\alpha_{3} w_{2}^{2}\right) \cos t \\
& \frac{d w_{2}}{d t}=\varepsilon^{2} w_{2}+\varepsilon \alpha_{4} w_{1} w_{2} \cos t
\end{aligned}
$$


Following the iteration procedure for equations (2) and (3) indicated in $\$ 1$ (in this example, $z$ is a vacuous component), we obtain the iterated-average system:

$$
\begin{aligned}
& \frac{d \rho_{1}}{d t}=\varepsilon^{2}\left\{\rho_{1}+\left(\alpha_{4}-\alpha_{1}\right) \rho_{1} \rho_{2}^{2}-\frac{1}{2} \alpha_{2} \rho_{2}^{3}\right\} \\
& \frac{d \rho_{2}}{d t}=\varepsilon^{2}\left(\rho_{2}-\frac{1}{2} \alpha_{4} \rho_{2}^{3}\right) .
\end{aligned}
$$

The $\left(\rho_{1}, \rho_{2}\right)$ equations have exponentially asymptotically stable rest points at $\left(\alpha_{2} \sqrt{2 / \alpha_{4}} /\left(3 \alpha_{4}-2 \alpha_{1}\right), \sqrt{2 / \alpha_{4}}\right)$ and at $\left(-\alpha_{2} \sqrt{2 / \alpha_{4}} /\left(3 \alpha_{4}-2 \alpha_{1}\right)\right.$, $\left.-\sqrt{2 / \alpha_{4}}\right)$ provided that $0<\alpha_{4}<2 \alpha_{1} / 3$. Therefore, in this case, $\S 1$ or the Theorem of $\S 4$ shows that for all small $\varepsilon>0$ and any $t_{0} \geq 0$ :

$$
\sup _{t_{0} \leq t<\infty}\left\{\left|w_{1}(t, \varepsilon)-\rho_{1}(t, \varepsilon)\right|+\left|w_{2}(t, \varepsilon)-\rho_{2}(t, \varepsilon)\right|\right\} \leq K^{*} \varepsilon
$$

where $K^{*}$ is independent of $\varepsilon, t_{0}$, and any initial value of $\left(w_{1}, w_{2}\right)$ selected from a fixed bounded domain of stability of the $\left(\rho_{1}, \rho_{2}\right)$ system. And if the inequality $0<\alpha_{4}<2 \alpha_{1} / 3$ does not hold, the approximation of $\left(w_{1}, w_{2}\right)$ by $\left(\rho_{1}, \rho_{2}\right)$ still holds for small $\varepsilon>0$ in the interval $t_{0} \leq t \leq$ $t_{0}+M / \varepsilon^{2}$ ( $M$ fixed but arbitrary).

Consequently, we see by the foregoing analysis that if $0<\alpha_{4}<2 \alpha_{1} / 3$, then two different solutions bifurcate from the origin of the $\left(x_{1}, x_{2}\right)$ system for all small $\varepsilon>0$. Their periodicity is easily established by the implicit function theorem, and their locations are given by

$$
\left(x_{1}, x_{2}\right)=\left(\frac{ \pm \varepsilon \alpha_{2} \sqrt{2 / \alpha_{4}}}{\left(3 \alpha_{4}-2 \alpha_{1}\right)}+O\left(\varepsilon^{2}\right), \pm \varepsilon \sqrt{2 / \alpha_{4}}+O\left(\varepsilon^{2}\right)\right) .
$$

And although it is clearly clear that each bifurcated solution captures all trajectories that get near it, the reader is referred to [10] for proof that these bifurcations are stable.

EXAMPLE 4. (Coupled Second Order Equations.) With $\alpha>0$ a rational constant and with $u$ and $x$ scalars, consider the system:

$$
\begin{aligned}
& \frac{d^{2} u}{d t^{2}}+u+\varepsilon^{2}\left[\beta_{1} u+\left(\beta_{2}+\beta_{3} u^{2}\right) \frac{d u}{d t}\right] \frac{d u}{d t}=0 \\
& \frac{d^{2} x}{d t^{2}}+\alpha^{2} x+\varepsilon\left(x^{2}-u^{2}\right) \frac{d x}{d t}=0
\end{aligned}
$$


which can be rewritten as:

$\frac{d}{d t}\left(\begin{array}{l}u \\ v \\ x \\ y\end{array}\right)=\left(\begin{array}{rrrr}0 & -1 & 0 & 0 \\ 1 & 0 & 0 & 0 \\ 0 & 0 & 0 & -\alpha \\ 0 & 0 & \alpha & 0\end{array}\right)\left(\begin{array}{l}u \\ v \\ x \\ y\end{array}\right)+\left(\begin{array}{c}0 \\ \varepsilon^{2}\left(\beta_{3} u^{2} v^{2}+\beta_{2} v^{2}-\beta_{1} u v\right) \\ 0 \\ \varepsilon\left(u^{2}-x^{2}\right) y\end{array}\right)$

Using

$$
\begin{aligned}
& u=w_{1} \cos \left(t+w_{3}\right), \\
& v=w_{1} \sin \left(t+w_{3}\right), \\
& x=w_{2} \cos \left(\alpha t+w_{4}\right), \\
& y=w_{2} \sin \left(\alpha t+w_{4}\right),
\end{aligned}
$$

we obtain:

$$
\begin{aligned}
\frac{d w_{1}}{d t}= & \varepsilon^{2} w_{1}^{2}\left\{\beta_{3} w_{1}^{2} \cos ^{2}\left(t+w_{3}\right)+\beta_{2}\right\} \sin ^{3}\left(t+w_{3}\right) \\
& -\varepsilon^{2} \beta_{1} w_{1}^{2} \sin ^{2}\left(t+w_{3}\right) \cos \left(t+w_{3}\right),
\end{aligned}
$$

$\frac{d w_{2}}{d t}=\varepsilon w_{2}\left\{w_{1}^{2} \cos ^{2}\left(t+w_{3}\right)-w_{2}^{2} \cos ^{2}\left(\alpha t+w_{4}\right)\right\} \sin ^{2}\left(\alpha t+w_{4}\right)$,

$\frac{d w_{3}}{d t}=\varepsilon^{2} w_{1}\left\{\beta_{3} w_{1}^{2} \cos ^{2}\left(t+w_{3}\right)+\beta_{2}\right\} \sin ^{2}\left(t+w_{3}\right) \cos \left(t+w_{3}\right)$

$$
-\varepsilon^{2} \beta_{1} w_{1} \cos ^{2}\left(t+w_{3}\right) \sin \left(t+w_{3}\right),
$$

$\frac{d w_{4}}{d t}=\varepsilon\left\{w_{1}^{2} \cos ^{2}\left(t+w_{3}\right)-w_{2}^{2} \cos ^{2}\left(\alpha t+w_{4}\right)\right\} \sin \left(\alpha t+w_{4}\right) \cos \left(\alpha t+w_{4}\right)$.

Then following the iteration procedure indicated in $\$ 1$ for equations (2) and (3) (here the $z$-equation is vacuous), we obtained the iterated average system:

$$
\begin{aligned}
& \frac{d \rho_{1}}{d t}=\varepsilon^{4} \bar{E}_{1,4}(\rho)=\frac{\varepsilon^{4}}{48} \beta_{1} \rho_{1}^{3}\left(6 \beta_{2}+\beta_{3} \rho_{1}^{2}\right), \\
& \frac{d \rho_{2}}{d t}=\varepsilon \bar{E}_{2,1}(\rho)=\frac{\varepsilon}{8} \rho_{2}\left(2 \rho_{1}^{2}-\rho_{2}^{2}\right), \\
& \frac{d \rho_{3}}{d t}=\varepsilon^{4} \bar{E}_{3,4}(\rho)=-\frac{\varepsilon^{4}}{24} \rho_{1}^{2}\left(\beta_{1}^{2}+4 \beta_{2}^{2}+5 \beta_{2} \beta_{3} \rho_{1}^{2}\right)-\frac{11 \varepsilon^{4}}{320} \beta_{3}^{2} \rho_{1}^{6}, \\
& \frac{d \rho_{4}}{d t}=\varepsilon \bar{E}_{4,1}(\rho)=0
\end{aligned}
$$

where $\rho=\left(\rho_{1}, \rho_{2}, \rho_{3}, \rho_{4}\right)$. Now restricting our attention to the $\rho_{+}$subsystem where $\rho_{+}=\left(\rho_{1}, \rho_{2}\right)$, we find an exponentially asymptotically 
stable rest point at $\left(\rho_{1}, \rho_{2}\right)=\left(\sqrt{-6 \beta_{2} / \beta_{3}}, \sqrt{-12 \beta_{2} / \beta_{3}}\right)$ provided $\beta_{1} \beta_{2}$ $>0$ and $\beta_{1} \beta_{3}<0$. The reader can check that Hypothesis $\mathrm{H} 4$ in $\S 3$ is satisfied. Consequently, if $\beta_{1} \beta_{2}>0$ and $\beta_{1} \beta_{3}<0$, then for any $t_{0} \geq 0$ and for all small $\varepsilon>0$,

$$
\sup _{t_{0} \leq t<\infty}\left\{\left|w_{1}(t, \varepsilon)-\rho_{1}(t, \varepsilon)\right|+\left|w_{2}(t, \varepsilon)-\rho_{2}(t, \varepsilon)\right|\right\} \leq K^{*} \varepsilon,
$$

where $K^{*}$ is independent of $\varepsilon, t_{0}$, and any initial value of $\left(w_{1}, w_{2}\right)$ selected from a fixed bounded domain of stability of the $\left(\rho_{1}, \rho_{2}\right)$ system. But when $\beta_{1} \beta_{2} \leq 0$ or $\beta_{1} \beta_{3} \geq 0$, Hypothesis H5 holds (provided both $\rho_{1}$ and $\rho_{2}$ are both positive at $t=t_{0}$ ) and the approximation is uniform on $t_{0} \leq t \leq t_{0}$ $+M / \varepsilon^{4}$ for any given $M>0$.

EXAMPLE 5. With $\alpha, \beta$, and $\gamma$ constant, consider the system:

$$
\begin{aligned}
& \frac{d w_{1}}{d t}=\varepsilon w_{1} \sin t+\varepsilon\left(\alpha w_{1}^{2}+\beta z_{2}^{2}\right) \cos 2 t, \\
& \frac{d w_{2}}{d t}=\varepsilon w_{2} z_{2} \sin t+\varepsilon \gamma z_{1} \cos 2 t, \\
& \frac{d w_{3}}{d t}=\varepsilon\left(w_{2}^{2}-w_{3}^{2}\right) \sin ^{2} t, \\
& \frac{d z_{1}}{d t}=-z_{1}+\varepsilon w_{2}^{3} \cos 2 t, \\
& \frac{d z_{2}}{d t}=-2 z_{2}+\varepsilon w_{1}^{2} \sin t .
\end{aligned}
$$

Using the iteration procedure outlined in $§ 1$, we obtain the iterated-average system:

$$
\begin{aligned}
\frac{d \rho_{1}}{d t} & =\frac{\varepsilon^{3}}{200} \rho_{1}^{2}\left(25 \alpha-6 \beta \rho_{1}^{2}\right), \\
\frac{d \rho_{2}}{d t} & =\frac{\varepsilon^{2}}{10} \rho_{2}\left(2 \rho_{1}^{2}+\gamma \rho_{2}^{2}\right), \\
\frac{d \rho_{3}}{d t} & =\frac{\varepsilon}{2}\left(\rho_{2}^{2}-\rho_{3}^{2}\right), \\
\frac{d \zeta_{1}}{d t} & =-\zeta_{1}, \\
\frac{d \zeta_{2}}{d t} & =-2 \zeta_{2} .
\end{aligned}
$$

With $\rho=\left(\rho_{1}, \rho_{2}, \rho_{3}\right)$, the $\rho$-system has two exponentially asymptotically stable rest points located at

$$
\left(\rho_{1}, \rho_{2}, \rho_{3}\right)=(\sqrt{25 \alpha /(6 \beta)}, \pm \sqrt{25 \alpha /(-3 \beta \gamma)}, \sqrt{25 \alpha /(-3 \beta \gamma)})
$$


provided $\alpha>0, \beta>0$, and $\gamma<0$. In this case, Hypothesis H4 of $\S 3$ holds for solutions to the $\rho$-system which start in the vicinity of either rest point. Consequently, when $\alpha>0, \beta>0$, and $\gamma<0$, then

$$
\sup _{t_{0} \leq t<\infty}\left\{\sum_{i=1}^{3}\left|w_{i}(t, \varepsilon)-\rho_{i}(t, \varepsilon)\right|+\sum_{i=1}^{2}\left|z_{i}(t, \varepsilon)-\zeta_{i}(t, \varepsilon)\right|\right\} \leq K^{*} \varepsilon
$$

for all small $\varepsilon \geq 0$ and any $t_{0} \geq 0$. The constant $K^{*}$ does not depend on $t_{0}$ or $\varepsilon$ or on which point in a fixed neighborhood of the rest point is selected for the value of $\rho$ at $t=t_{0}$. Moreover, if $\gamma<0$ but not both $\alpha>0$ and $\beta>0$, then the approximation is uniform on intervals $t_{0} \leq t \leq t_{0}+M / \varepsilon^{3}$, where the value of $\rho$ at $t=t_{0}$ and the fixed value of $M(>0)$ are chosen such that Hypothesis $\mathrm{H} 5$ holds.

By the implicit function theorem, many of the rest points of the $(\rho, \zeta)$ system correspond (approximately) to locations of periodic solutions for the $(w, z)$ system. The reader is referred to [11] to determine the stability of these periodic solutions provided we have the condition $\gamma<0$ and $\alpha \beta>0$.

3. Hypotheses. With the integers $l, l^{*}$, and $m$ from $\S 1$, we recall that $w_{+}=\left(w_{1}, w_{2}, \ldots, w_{l}\right)$ and $w=\left(w_{1}, w_{2}, \ldots, w_{l^{*}}\right)$, where $l \leq l^{*}$, and $z$ lies in $R^{m}$. Define $l_{0}=l^{*}-l$. Next let $D_{+}$and $D_{z}$ be bounded convex open sets in $R^{l}$ and $R^{m}$, respectively; let $S_{+}$and $S_{z}$ be open sets with closures contained in $D_{+}$and $D_{z}$, respectively; and let $\dot{S}_{+}^{\circ}$ and $S_{z}^{\circ}$ be respective subsets of $S_{+}$and $S_{z}$. Then for some $\varepsilon_{D}>0$ define the set $D$ consisting of points of the form $(w, z, t, \varepsilon)$ by $D=\left(D_{+} \times R^{l_{0}}\right) \times D_{z} \times$ $[0, \infty) \times\left[0, \varepsilon_{D}\right]$. Regarding (1a), (1b), we assume

Hypothesis H1 (Periodicity, Smoothness). Each $E_{i}(w, z, t, \varepsilon)$, for $1 \leq i \leq l^{*}$, and $H(w, z, t, \varepsilon)$ are periodic in $t$ with a fixed common period $P>0$. Moreover, each $E_{l}$ and $H$ and several orders of their derivatives with respect to $(w, z, \varepsilon)$ are uniformly bounded on the set $D$, and for each fixed $t \geq 0$, are smooth functions of $(w, z, \varepsilon)$ on $\left(D_{+} \times R^{l_{0}}\right) \times D_{z} \times\left[0, \varepsilon_{D}\right]$. Finally, constants $\delta>0$ and $K_{A}>0$ exist such that for all $t \geq 0$, the constant matrix $A$ satisfies $\left\|e^{A t}\right\| \leq K_{A} e^{-\delta t}$, where \|\| is the matrix norm.

With $t_{0}=t_{0}(\varepsilon)$ an initial point either depending on $\varepsilon$ or simply constant, assume system (1a), (1b) satisfies the initial conditions:

$$
\begin{aligned}
\left.w_{l}\right|_{t=t_{0}(\varepsilon)} & =b_{0 l}+\varepsilon b_{1 i}(\varepsilon), \quad 1 \leq i \leq l^{*}, \\
\left.z\right|_{t=t_{0}(\varepsilon)} & =\xi_{0}+\varepsilon \xi_{1}(\varepsilon),
\end{aligned}
$$

and let $b_{0}=\left(b_{01}, b_{02}, \ldots, b_{0 l^{*}}\right)$ and $b_{1}(\varepsilon)=\left(b_{11}(\varepsilon), b_{12}(\varepsilon), \ldots, b_{11^{*}}(\varepsilon)\right)$. 
HyPothesis H2 (Initial Data). $\left(b_{0}, \xi_{0}\right)$ lies in $\left(\dot{S}_{+} \times R^{l_{0}}\right) \times \dot{S}_{z}$, and on $0 \leq \varepsilon \leq \varepsilon_{D},\left|\left(b_{1}(\varepsilon), \xi_{1}(\varepsilon)\right)\right| \leq N_{0}$ for some fixed constant $N_{0}$.

Defining the iterated-averages $\bar{E}_{i, n_{l}}(\rho)$ as indicated in $\S 1$, and setting $s$ equal to $t_{0}(\varepsilon)$ in them if they depend on $s$ explicitly, consider:

Hypothesis H3 (The Iterated-Average System). Assume the $\bar{E}_{i, n_{i}}$ for $1 \leq i \leq l$ are independent of $\left(\rho_{l+1}, \rho_{l+2}, \ldots, \rho_{l^{*}}\right)$, so that we may write $\bar{E}_{i, n_{l}}=\bar{E}_{i, n_{1}}\left(\rho_{+}\right)$, where $\rho_{+}=\left(\rho_{1}, \rho_{2}, \ldots, \rho_{l}\right)$. Then formulating system $(5 \mathrm{a}),(5 \mathrm{~b})$, suppose further that

$$
\begin{aligned}
\left.\rho_{i}\right|_{t=t_{0}(\varepsilon)} & =b_{0 i}, \quad 1 \leq i \leq l, \\
\left.\zeta\right|_{t=t_{0}(\varepsilon)} & =\xi_{0}
\end{aligned}
$$

is satisfied. Defining $n=\max _{1 \leq l \leq l} n_{i}$, assume, finally, that a constant $M>0$ exists $(M$ may be $\infty)$ such that the solution $\left(\rho_{+}, \zeta\right)=$ $\left(\rho_{+}(t, \varepsilon), \zeta(t, \varepsilon)\right)$, to the initial value problem (5a), (5b) and (7a), (7b), exists and remains in $S_{+} \times S_{z}$ for $t_{0}(\varepsilon) \leq t \leq t_{0}(\varepsilon)+M / \varepsilon^{n}$ and $0<\varepsilon \leq \varepsilon_{D}$, and for all choices of $\left(b_{01}, b_{02}, \ldots, b_{0 l}, \xi_{0}\right)$ in $\dot{S}_{+} \times \dot{S}_{z}$.

We now let the matrix $U(t, \tau)$ with scalar entries $U_{i j}(t, \tau)$ be the fundamental solution to

$$
\frac{d U_{i j}}{d t}=\left.\sum_{k=1}^{l} \varepsilon^{n_{\imath}} \frac{\partial \bar{E}_{i, n_{t}}\left(\rho_{+}\right)}{\partial \rho_{k}}\right|_{\rho_{+}=\rho(t, \varepsilon)} \times U_{k j},\left.\quad U_{t j}\right|_{t=\tau}=\delta_{\imath j},
$$

for $1 \leq i \leq l, 1 \leq j \leq l$, with $\rho_{+}(t, \varepsilon)$ as described in Hypothesis H3 and with $\delta_{i j}$ the Kronecker delta. This system will be required either to be exponentially asympotically stable with respect to $U=0$, or else to have a limitation on the rate of growth of its solution.

Hypothesis H4 (Stability of the Variational Systems). Assume $M=$ $\infty$. Then constants $K_{E}, \lambda_{1}, \lambda_{2}, \ldots, \lambda_{l}$ (all $>0$ ) are assumed to exist independent of $\varepsilon, t_{0}(\varepsilon), \tau$, and any $\rho_{+}(t, \varepsilon)$ chosen in Hypothesis $\mathrm{H} 3$ such that for $t_{0}(\varepsilon) \leq \tau \leq t<\infty$, for $0<\varepsilon \leq \varepsilon_{D}$, and for all chosen $\rho_{+}$,

$$
\left\|U_{i j}(t, \tau)\right\| \leq K_{E} \sum_{\nu=1}^{l} \varepsilon^{\max \left(n_{\nu}-n_{\jmath}, 0\right)} e^{-\varepsilon^{n_{\nu}} \lambda_{\nu}(t-\tau)},
$$

where $1 \leq i \leq l$ and $1 \leq j \leq l$.

The above hypothesis has been formulated in very general fashion. However, simplification takes place in a great many applications. For 
example, when all the $n_{l}$ for $i \leq l$ are equal, with say, $n_{l}=n$, then the required variational inequality becomes

$$
\left\|U_{i j}(t, \tau)\right\| \leq K_{E} l e^{-\varepsilon^{n}(t-\tau) \min _{\nu} \lambda_{\nu}},
$$

and determining whether this can be satisfied is routine.

And now we provide for systems that lack total stability.

HyPOTHESIS H5 (Limitation of Growth). Assume $M<\infty$ and $n_{1} \leq n_{2}$ $\leq \cdots \leq n_{c-1}<n_{c}=n_{c+1}=\cdots=n_{l-1}=n_{l}(=n)$ where $c \leq l$. Then constants $K_{E}(M), \lambda_{1}(M), \lambda_{2}(M), \ldots, \lambda_{c-1}(M)$, and $N_{l}(M)$ (all $>0$ ) are assumed to exist independent of $\varepsilon, \tau, t_{0}(\varepsilon)$, and any $\rho_{+}(t, \varepsilon)$ chosen in Hypothesis H3, such that for $t_{0}(\varepsilon) \leq \tau \leq t \leq t_{0}(\varepsilon)+M / \varepsilon^{n}$, for $0<\varepsilon \leq$ $\varepsilon_{D}$, and for all chosen $\rho_{+}$,

$$
\begin{aligned}
\left\|U_{i j}(t, \tau)\right\| \leq & K_{E}(M) \sum_{\nu=1}^{c-1} \varepsilon^{\max \left(n_{\nu}-n_{\jmath}, 0\right)} e^{-\varepsilon^{n_{\nu}} \lambda_{\nu}(M)(t-\tau)} \\
& +K_{E}(M) \varepsilon^{n-n_{J}} e^{\varepsilon^{n} N_{l}(M)(t-\tau)}
\end{aligned}
$$

where $1 \leq i \leq l$ and $1 \leq j \leq l$.

The preceding hypothesis is particularly simple to verify when all the $n_{i}$ for $i \leq l$ are equal (the case with $c=1$ ), as our inequality becomes merely

$$
\|U(t, \tau)\| \leq K_{E}(M) e^{\varepsilon^{n} N_{t}(M)(t-\tau)} .
$$

In other cases the reader may have to renumber his components in order to be consistent with the format of Hypothesis H5 (see Example 4).

\section{The main result.}

THEOREM. Let $(w(t, \varepsilon), z(t, \varepsilon))$ be a solution to the initial value problem (1a), (1b), (6a), (6b), with $t_{0}(\varepsilon) \geq 0$ arbitrarily chosen, and let $\left(\rho_{+}(t, \varepsilon)\right.$, $\zeta(t, \varepsilon)$ ) be a solution to the multi-scale averaged system $(5 \mathrm{a}),(5 \mathrm{~b}),(7 \mathrm{a}),(7 \mathrm{~b})$, for $0<\varepsilon \leq \varepsilon_{D}$. Let

$$
w_{+}(t, \varepsilon)=\left(w_{1}(t, \varepsilon), w_{2}(t, \varepsilon), \ldots, w_{l}(t, \varepsilon)\right) .
$$

Case A. Let Hypotheses $\mathrm{H1}-\mathrm{H} 4$ hold $(M=\infty)$. Then constants $K^{*}$, $\varepsilon^{*}>0$ exist (with values independent of $t_{0}(\varepsilon)$, but depending on $D, S_{+}$, $S_{z}, \dot{S}_{+}, \dot{S}_{z}$, and the bounds in H1-H4) such that for $0<\varepsilon<\varepsilon^{*}$ the solutions $(w(t, \varepsilon), z(t, \varepsilon))$ and $\left(\rho_{+}(t, \varepsilon), \zeta(t, \varepsilon)\right)$ exist on $t_{0}(\varepsilon) \leq t<\infty$, 
and

$$
\sup _{t_{0}(\varepsilon) \leq t<\infty}\left\{\left|w_{+}(t, \varepsilon)-\rho_{+}(t, \varepsilon)\right|+|z(t, \varepsilon)-\zeta(t, \varepsilon)|\right\} \leq K^{*} \varepsilon
$$

uniformly for initial values $\left(b_{0}, \xi_{0}\right)$ in $\left(\stackrel{\circ}{S}_{+} \times R^{I_{0}}\right) \times \stackrel{\circ}{S}_{z}$.

Case B. Let Hypothesis $\mathrm{H} 1-\mathrm{H} 3$ and $\mathrm{H} 5$ hold with $M>0$ finite. Then constants $K_{n}(M), \varepsilon_{n}(M)>0$ exist (with values independent of $t_{0}(\varepsilon)$, but depending on $D, S_{+}, S_{z}, \dot{S}_{+}, \dot{S}_{z}$, and the bounds in $\mathrm{H} 1-\mathrm{H} 3$ and $\mathrm{H} 5)$ such that for $0 \leq \varepsilon \leq \varepsilon_{n}(M)$, the solutions $(w(t, \varepsilon), z(t, \varepsilon))$ and $\left(\rho_{+}(t, \varepsilon), \zeta(t, \varepsilon)\right)$ exist on $t_{0}(\varepsilon) \leq t \leq t_{0}(\varepsilon)+M / \varepsilon^{n}$, and

$$
\sup _{t_{0}(\varepsilon) \leq t \leq t_{0}(\varepsilon)+M / \varepsilon^{n}}\left\{\left|w_{+}(t, \varepsilon)-\rho_{+}(t, \varepsilon)\right|+|z(t, \varepsilon)-\zeta(t, \varepsilon)|\right\} \leq K_{n}(M) \varepsilon
$$

uniformly for initial values $\left(b_{0}, \xi_{0}\right)$ in $\left(\stackrel{\circ}{S}_{+} \times R^{l_{0}}\right) \times \stackrel{\circ}{S}_{z}$.

Consequently, the theorem states that if $E_{l}(w, z, t, \varepsilon)$ from (1a), (1b) is expanded in powers of $\varepsilon$, with coefficients in both $t$ and the stroboscopic projection of $w$ (the variable $z$ is eventually set to zero), then $E_{l}$ may be replaced by its first nonzero average $\varepsilon^{n_{l}-1} \bar{E}_{l, n_{l}}$ in the expansion to obtain the approximating system. This result applies to a greater variety of systems than the theorem in [9], because here motion may be carried on several characteristic scales at the same time, and the averages $\bar{E}_{l, n_{l}}$ are allowed to depend on the choice of the initial point $t=t_{0}(\varepsilon)$.

5. Proof of the theorem. With $(w, z)=(w(t, \varepsilon), z(t, \varepsilon))$ and $\left(\rho_{+}, \zeta\right)$ $=\left(\rho_{+}(t, \varepsilon), \zeta(t, \varepsilon)\right)$ the respective solutions to (1a), (1b), (6a), (6b) and to (5a), (5b), (7a), (7b), let $w_{t}=\rho_{l}+\varepsilon W_{i}(1 \leq i \leq l)$ and $z=\zeta+\varepsilon Z$. Let $d$ be the distance between the boundaries of $S_{+} \times S_{z}$ and $D_{+} \times D_{z}$, and let $N_{1}$ majorize $A, E_{l}(w, z, t, \varepsilon)$ (for $\left.1 \leq i \leq l^{*}\right), H(w, z, t, \varepsilon)$, and their appropriate derivatives on the set $D$, and majorize $\left(b_{11}(\varepsilon)\right.$, $\left.b_{12}(\varepsilon), \ldots, b_{11^{*}}(\varepsilon), \xi_{1}(\varepsilon)\right)$ on $0 \leq \varepsilon \leq \varepsilon_{D}$. We now can write

$$
\begin{aligned}
& (8 \mathrm{a}, \mathrm{b}) \frac{d W_{i}}{d t}=E_{l}(w, z, t, \varepsilon)-\varepsilon^{n_{t}-1} \bar{E}_{l, n_{t}}\left(\rho_{+}\right),\left.\quad W_{\imath}\right|_{t=t_{0}(\varepsilon)}=b_{1_{l}}(\varepsilon), \\
& \text { (9a,b) } \frac{d Z}{d t}=A Z+H(w, z, t, \varepsilon),\left.\quad Z\right|_{t=t_{0}(\varepsilon)}=\xi_{1}(\varepsilon) .
\end{aligned}
$$

Now for any constant

$$
a>\sup _{0<\varepsilon \leq \varepsilon_{D}}\left\{\left|b_{11}(\varepsilon)\right|,\left|b_{12}(\varepsilon)\right|, \ldots,\left|b_{1 /}(\varepsilon)\right|,\left|\xi_{1}(\varepsilon)\right|\right\},
$$


there exists $t_{1}(\varepsilon)>t_{0}(\varepsilon),\left(t_{1}(\varepsilon) \leq t_{0}(\varepsilon)+M / \varepsilon^{n}\right)$ such that $(w(t, \varepsilon), z(t, \varepsilon))$ exists on $t_{0}(\varepsilon) \leq t \leq t_{1}(\varepsilon)$ for $0<\varepsilon \leq \varepsilon_{D}$, and

$$
\sup _{t_{0}(\varepsilon) \leq t \leq t_{1}(\varepsilon)}\left\{\left|W_{1}(t, \varepsilon)\right|,\left|W_{2}(t, \varepsilon)\right|, \ldots,\left|W_{l}(t, \varepsilon)\right|,|Z(t, \varepsilon)|\right\} \leq a
$$

for $0<\varepsilon \leq \varepsilon_{D}$. Choosing $\varepsilon_{1}=\min \left(\varepsilon_{D}, d /(2 a), 1\right)$, then by Hypothesis $\mathrm{H} 3$, $(w(t, \varepsilon), z(t, \varepsilon))$ lies in $\left(D_{+} \times R^{l_{0}}\right) \times D_{z}$ for $t_{0}(\varepsilon) \leq t \leq t_{1}(\varepsilon), 0<\varepsilon<\varepsilon_{1}$. Now from $(9 a),(9 b)$ we obtain

$$
\begin{aligned}
Z(t)= & V\left(t, t_{0}(\varepsilon)\right) \xi_{1}(\varepsilon) \\
& +\int_{t_{0}(\varepsilon)}^{t} V(t, \tau) H(w(\tau), z(\tau), \tau, \varepsilon) d \tau
\end{aligned}
$$

where $V(t, \tau) \equiv e^{A(t-\tau)}, w(t) \equiv w(t, \varepsilon), z(t) \equiv z(t, \varepsilon)$, etc. Then by Hypotheses $\mathrm{H} 1$ and $\mathrm{H} 2,|Z(t)| \leq N_{1}\left(1+K_{A} / \delta\right)$ for $t_{0}(\varepsilon) \leq t \leq t_{1}(\varepsilon)$ and $0<\varepsilon \leq \varepsilon_{1}$, independent of $\varepsilon, a, t_{0}(\varepsilon), t_{1}(\varepsilon)$ and not depending on the chosen value of $\left.(w, z)\right|_{t=t_{0}(\varepsilon)}$.

\section{Expansion of the $E_{i}(w, z, t, \varepsilon)$}

With $t_{0}(\varepsilon) \leq s \leq t$, consider the equations

$$
\begin{aligned}
w_{i}(t) & =w_{l}(s)+\varepsilon \int_{s}^{t} E_{i}(w(\tau), z(\tau), \tau, \varepsilon) d \tau, \quad 1 \leq i \leq l^{*}, \\
z(t) & =z^{(0)}(t)+\varepsilon \int_{t_{0}(\varepsilon)}^{t} V(t, \tau) H(w(\tau), z(\tau), \tau, \varepsilon) d \tau
\end{aligned}
$$

obtained earlier. Using Taylor's theorem, we now expand each $E_{l}$ and the vector $H$ about the point $(w, z, t, \varepsilon)=(w(s), 0, t, 0)$. Then repeated iteration of (2) and (3) leads to

$$
\begin{aligned}
E_{l}(w(t), z(t), t, \varepsilon)= & \sum_{J=1}^{n_{l}} \varepsilon^{J-1} E_{i, j}\left(w(s), t, s, t_{0}(\varepsilon)\right) \\
& +R_{\iota, n_{l}}\left(w(t), z(t), t, \varepsilon, s, t_{0}(\varepsilon)\right)
\end{aligned}
$$

for $1 \leq i \leq l^{*}$, where the $E_{l, j}$ depend on the initial points $s$ and $t_{0}(\varepsilon)$, but not on $\varepsilon$ (except through $t_{0}(\varepsilon)$ ) or on $z^{(0)}(t)$. The terms involving $z^{(0)}(t)$ have been merged into the remainder $R_{i, n_{i}}$. And from Hypothesis $\mathrm{H} 2$,

$$
\left|z^{(0)}(t)\right| \leq K_{A}\left(\left|\xi_{0}\right|+\varepsilon\left|\xi_{1}(\varepsilon)\right|\right) e^{-\delta\left(t-t_{0}(\varepsilon)\right)} .
$$

Moreover, for $t_{0}(\varepsilon) \leq s \leq t \leq t_{1}(\varepsilon)$ and $0<\varepsilon \leq \varepsilon_{1},(w(t), z(t))$ lies in $\left(D_{+} \times R^{I_{0}}\right) \times D_{z}$, and with $Q$ representing any $E_{l}$ or $H$ or any of their 
derivatives used in obtaining the $E_{i, j}$ and the $R_{i, n_{l}}$, we have

$$
\begin{gathered}
|Q(w(t), z(t), t, \varepsilon)-Q(w(s), 0, t, 0)| \\
\leq N_{1}(|w(t)-w(s)|+|z(t)|+\varepsilon) .
\end{gathered}
$$

Moreover, for $t_{0}(\varepsilon) \leq s \leq t \leq t_{1}(\varepsilon)$,

$$
\begin{aligned}
\mid w(t) & -w(s)|+| z(t) \mid+\varepsilon \\
\leq & \varepsilon\left(1+\frac{N_{1} K_{A}}{\delta}+N_{1}|t-s|\right)+\left|z^{(0)}(t)\right| \\
\leq & \varepsilon\left(1+\frac{N_{1} K_{A}}{\delta}+N_{1}\right)(1+|t-s|) \\
& +K_{A}\left(\left|\xi_{0}\right|+\varepsilon\left|\xi_{1}(\varepsilon)\right|\right) e^{-\delta\left(s-t_{0}(\varepsilon)\right)} \\
\leq & N_{10}(1+|t-s|)\left(\varepsilon+e^{-\delta\left(s-t_{0}(\varepsilon)\right)}\right)
\end{aligned}
$$

where $N_{10}$ is independent of $t_{0}(\varepsilon), t_{1}(\varepsilon), \varepsilon$, and $a$, provided $0<\varepsilon \leq \varepsilon_{1}$. Note that

$$
z^{(0)}(t) \leq N_{10}(1+|t-s|)\left(\varepsilon+e^{-\delta\left(s-t_{0}(\varepsilon)\right)}\right),
$$

as well. Therefore,

$$
\begin{aligned}
& \{|w(t)-w(s)|+|z(t)|+\varepsilon\}^{J}\left\{z^{(0)}(t)\right\}^{\mu-j} \\
& \quad \leq N_{10}^{\mu}(1+|t-s|)^{\mu}\left(\varepsilon+e^{-\delta\left(s-t_{0}(\varepsilon)\right)}\right)^{\mu} \\
& \quad \leq N_{10}^{\mu}(1+|t-s|)^{\mu}\left(\varepsilon^{\mu}+e^{-\delta\left(s-t_{0}(\varepsilon)\right)}\right)(\mu+1) !
\end{aligned}
$$

for any integers $\mu \geq 1, j \leq \mu(j \geq 0)$. For $t_{0}(\varepsilon) \leq s \leq t$, we have:

$$
\begin{aligned}
& \int_{s}^{t}(1+|\tau-s|)^{\mu} d \tau \leq \frac{1}{\mu+1}(1+|t-s|)^{\mu+1} \\
& \int_{t_{0}(\varepsilon)}^{t}\|V(t, \tau)\|(1+|\tau-s|)^{\mu} d \tau \\
& \leq \frac{K_{A}}{\mu+1}(1+|t-s|)^{\mu+1}+K_{A} \int_{t_{0}(\varepsilon)}^{s} e^{-\delta(t-\tau)}(1+|\tau-s|)^{\mu} d \tau \\
& \leq \frac{K_{A}}{\mu+1}(1+|t-s|)^{\mu+1}+\frac{K_{A}}{\delta} e^{-\delta(t-s)}(\mu+1)\left(1+\frac{\mu}{\delta}\right)^{\mu+1} \\
& \leq N_{11}(\mu)(1+|t-s|)^{\mu+1}
\end{aligned}
$$


where $N_{11}(\mu)$ is constant. Therefore, employing (2), (3), (12)-(16), and the bounds on $z^{(0)}(t)$ in deriving equation (11), we find that for $t_{0}(\varepsilon) \leq s \leq t$ $\leq t_{1}(\varepsilon)$ and $0<\varepsilon \leq \varepsilon_{1}$,

$$
\begin{aligned}
& \left|R_{t, n_{t}}\left(w(t), z(t), t, \varepsilon, s, t_{0}(\varepsilon)\right)\right| \\
& \quad \leq N_{12}(1+|t-s|)^{\beta}\left(\varepsilon^{n_{t}}+e^{-\delta\left(s-t_{0}(\varepsilon)\right)}\right)
\end{aligned}
$$

where $\beta$ and $N_{12}$ are positive constants independent of $\varepsilon, a, t_{0}(\varepsilon), t_{1}(\varepsilon)$, and $i$.

\section{The Expanded $W_{l}$-Equations}

Now letting $\Gamma[]$ be the greatest integer function, define the step function $\hat{t}$ by $\hat{t}=t_{0}(\varepsilon)+P \times \Gamma\left[\left(t-t_{0}(\varepsilon)\right) / P\right]$, where $P$ is the period in Hypothesis H1. Since $s$ in (11) was unspecified, let $s=\hat{t}$ so $w(s)=w(\hat{t})$ there. Writing $\hat{w}$ or $\hat{w}(t)$ for $w(\hat{t})$ and substituting (11) into (8a), we obtain

$$
\begin{aligned}
\frac{d W_{i}}{d t}= & \sum_{J=1}^{n_{t}} \varepsilon^{J-1} E_{t, j}\left(\hat{w}, t, \hat{t}, t_{0}(\varepsilon)\right)-\varepsilon^{n_{\iota}-1} \bar{E}_{t, n_{\imath}}\left(\rho_{+}\right) \\
& +R_{i, n_{\imath}}\left(w, z, t, \varepsilon, \hat{t}, t_{0}(\varepsilon)\right), \quad\left(1 \leq i \leq i_{E}\right) .
\end{aligned}
$$

Moreover, since $s=\hat{t}$ and $0 \leq t-\hat{t} \leq P$, then from (17) a constant $N_{2}$ exists independent of $\varepsilon, a, t_{0}(\varepsilon), t_{1}(\varepsilon)$, and $i$, such that

$$
\left|R_{i, n_{t}}\left(w(t), z(t), t, \varepsilon, \hat{t}, t_{0}(\varepsilon)\right)\right| \leq N_{2}\left(\varepsilon^{n_{t}}+e^{-\delta\left(t-t_{0}(\varepsilon)\right) / 2}\right)
$$

provided $t_{0}(\varepsilon) \leq t \leq t_{1}(\varepsilon)$ and $0<\varepsilon \leq \varepsilon_{1}$.

Since by $\S 1$, the averages of $\left.E_{l, j}\left(\rho, t, s, t_{0}(\varepsilon)\right)\right|_{t_{0}=-\infty}$ are zero for $1 \leq i \leq l, 1 \leq j \leq n_{l}-1$, we can define $\bar{E}_{l, j} \equiv 0$ in these cases. Moreover, for $1 \leq i \leq l$ and for $w$ in $D_{+} \times R^{l_{0}}, \bar{E}_{i, n_{l}}(w)$ is well defined and equals $\bar{E}_{l, n_{l}}\left(w_{+}\right)$by Hypothesis H3. With $W_{+} \stackrel{2,}{=}\left(W_{1}, W_{2}, \ldots, W_{l}\right)$ and $w_{+}=\rho_{+}$ $+\varepsilon W_{+}$, equation (18) becomes

$$
\begin{aligned}
\frac{d W_{l}}{d t}= & \sum_{j=1}^{n_{l}} \varepsilon^{j-1}\left\{E_{l, j}\left(\hat{w}, t, \hat{t}, t_{0}(\varepsilon)\right)-E_{l, j}(\hat{w}, t, \hat{t},-\infty)\right\} \\
& +\sum_{j=1}^{n_{l}} \varepsilon^{j-1}\left\{E_{l, j}(\hat{w}, t, \hat{t},-\infty)-\bar{E}_{l, j}(\hat{w})\right\} \\
& +\varepsilon^{n_{l}-1}\left\{\bar{E}_{l, n_{l}}(\hat{w})-\bar{E}_{l, n_{l}}(w)\right\} \\
& +\varepsilon^{n_{l}-1}\left\{\bar{E}_{l, n_{l}}\left(\rho_{+}+\varepsilon W_{+}\right)-\bar{E}_{l, n_{l}}\left(\rho_{+}\right)\right\} \\
& +R_{l, n_{l}}\left(w, z, t, \varepsilon, \hat{t}, t_{0}(\varepsilon)\right) .
\end{aligned}
$$


Hence with $\varepsilon_{2}=\min \left(\varepsilon_{1}, 1 / a^{2}\right)$, we have

$$
\begin{aligned}
\frac{d W_{l}}{d t}= & \sum_{j=1}^{n_{l}} \varepsilon^{j-1}\left\{E_{l, j}(\hat{w}, t, \hat{t},-\infty)-\bar{E}_{i, j}(\hat{w})\right\} \\
& +\left.\varepsilon^{n_{i}} \sum_{j=1}^{n_{t}} \frac{\partial \bar{E}_{i, n_{i}}\left(w_{+}\right)}{\partial w_{j}}\right|_{w_{+}=\rho_{+}} W_{j}+C_{i}\left(W_{+}, Z, t, \varepsilon\right)
\end{aligned}
$$

where a constant $N_{3}$ exists independent of $\varepsilon, a, t_{0}(\varepsilon), t_{1}(\varepsilon)$, and $i$ such that

$$
\begin{aligned}
\left|C_{i}\left(W_{+}(t), Z(t), t, \varepsilon\right)\right| & \leq N_{3}\left(\varepsilon^{n_{\imath}}+\varepsilon^{n_{l}+1} a^{2}+e^{-\delta\left(t-t_{0}(\varepsilon)\right) / 2}\right) \\
& \leq N_{3}\left(2 \varepsilon^{n_{\imath}}+e^{-\delta\left(t-t_{0}(\varepsilon)\right) / 2}\right),
\end{aligned}
$$

for $0<\varepsilon \leq \varepsilon_{2}, t_{0}(\varepsilon) \leq t \leq t_{1}(\varepsilon), 1 \leq i \leq l$. Using $U(t, s)$ as defined preceding Hypothesis $\mathrm{H} 4,(8 \mathrm{~b})$ and (20) may be written as

$$
\begin{aligned}
W_{\nu}(t)= & \sum_{i=1}^{l} U_{\nu i}\left(t, t_{0}(\varepsilon)\right) b_{1 i}(\varepsilon) \\
& +\sum_{i=1}^{l} \sum_{j=1}^{n_{l}} \varepsilon^{j-1} \int_{t_{0}(\varepsilon)}^{t} U_{\nu i}(t, \tau) \\
& \quad \times\left\{E_{i, j}(\hat{w}(\tau), \tau, \hat{\tau},-\infty)-\bar{E}_{l, j}(\hat{w}(\tau))\right\} d \tau \\
+ & \sum_{i=1}^{l} \int_{t_{0}(\varepsilon)}^{t} U_{\nu i}(t, \tau) C_{i}\left(W_{+}(\tau), Z(\tau), \tau, \varepsilon\right) d \tau \quad \quad(1 \leq \nu \leq l),
\end{aligned}
$$

with $\hat{\tau}$ and $\hat{w}(\tau)$ defined like $\hat{t}$ and $\hat{w}(t)$. Noting that

$$
U(t, \tau)=U\left(t, t_{0}(\varepsilon)\right) U^{-1}\left(\tau, t_{0}(\varepsilon)\right)
$$

and

$$
\frac{d U^{-1}\left(\tau, t_{0}(\varepsilon)\right)}{d \tau}=-U^{-1}\left(\tau, t_{0}(\varepsilon)\right) \frac{d U\left(\tau, t_{0}(\varepsilon)\right)}{d \tau} U^{-1}\left(\tau, t_{0}(\varepsilon)\right)
$$


we use integration by parts in equation (21) to obtain (for $1 \leq \nu \leq l$ )

$$
\begin{aligned}
W_{\nu}(t)= & \sum_{k=1}^{l} U_{\nu k}\left(t, t_{0}(\varepsilon)\right) b_{1 k}(\varepsilon) \\
& +\sum_{j=1}^{n_{\nu}} \varepsilon^{j-1} \int_{t_{0}(\varepsilon)}^{t}\left\{E_{\nu, j}(\hat{w}(\tau), \tau, \hat{\tau},-\infty)-\bar{E}_{\nu, j}(\hat{w}(\tau))\right\} d \tau \\
& +\left.\sum_{i, k=1}^{l} \int_{t_{0}(\varepsilon)}^{t} U_{\nu k}(t, \tau) \varepsilon^{n_{k}} \frac{\partial \bar{E}_{k, n_{k}}\left(\rho_{+}\right)}{\partial \rho_{i}}\right|_{\rho_{+}=\rho(t, \varepsilon)} \\
& \times\left[\sum_{j=1}^{n_{t}} \varepsilon^{j-1} \int_{t_{0}(\varepsilon)}^{\tau}\left\{E_{i, j}(\hat{w}(\sigma), \sigma, \hat{\sigma},-\infty)-\bar{E}_{l, j}(\hat{w}(\sigma))\right\} d \sigma\right] d \tau \\
& +\sum_{k=1}^{l} \int_{t_{0}(\varepsilon)}^{t} U_{\nu k}(t, \tau) C_{k}\left(W_{+}(\tau), Z(\tau), \tau, \varepsilon\right) d \tau .
\end{aligned}
$$

Now a constant $N_{4}$ exists independent of $\varepsilon, a, t_{0}(\varepsilon), t_{1}(\varepsilon), i, j, k$, that majorizes $\partial \vec{E}_{k, n_{k}}\left(\rho_{+}\right) / \partial \rho_{i}$ on $D_{+}$, and by Hypotheses $\mathrm{H} 1$ and $\mathrm{H} 3$, majorizes

$$
\int_{t_{0}(\varepsilon)}^{t}\left\{E_{i, j}(\hat{w}(\tau), \tau, \hat{\tau},-\infty)-\bar{E}_{i, j}(\hat{w}(\tau))\right\} d \tau
$$

for $0<\varepsilon \leq \varepsilon_{2}, t_{0}(\varepsilon) \leq t \leq t_{1}(\varepsilon), 1 \leq i \leq l, 1 \leq j \leq n_{i}, 1 \leq k \leq l$. Then for $0<\varepsilon \leq \varepsilon_{2}, t_{0}(\varepsilon) \leq t \leq t_{1}(\varepsilon)$,

$$
\begin{aligned}
\left|W_{\nu}(t)\right| \leq & \sum_{k=1}^{l}\left\|U_{\nu k}\left(t, t_{0}(\varepsilon)\right)\right\| N_{1}+N_{4} \sum_{j=1}^{n_{\nu}} \varepsilon^{j-1} \\
& +\sum_{k=1}^{l} \int_{t_{0}(\varepsilon)}^{t}\left\|U_{\nu k}(t, \tau)\right\| \\
& \times\left\{\varepsilon^{n_{k}} N_{4}^{2} \sum_{i=1}^{l} \sum_{j=1}^{n_{l}} \varepsilon^{j-1}+N_{3}\left(2 \varepsilon^{n_{k}}+e^{-\delta\left(\tau-t_{0}(\varepsilon)\right) / 2}\right)\right\} d \tau
\end{aligned}
$$

with $1 \leq \nu \leq l$. The remainder of the proof will now be split into two cases.

The rest of the proof of case A $(M=\infty)$. Now from Hypothesis H4, constants $K_{E}, \lambda_{i}>0$ (for $1 \leq i \leq l$ ) exist such that

$$
\left\|U_{\nu k}(t, \tau)\right\| \leq K_{E} \sum_{i=1}^{l} \varepsilon^{\max \left(n_{i}-n_{k}, 0\right)} e^{-\varepsilon^{n_{t}} \lambda_{l}(t-\tau)}
$$


for all $t_{0}(\varepsilon) \leq \tau \leq t<\infty, 0<\varepsilon \leq \varepsilon_{D}$, independent of $t_{0}(\varepsilon) \geq 0$, and the chosen $\rho_{+}(t, \varepsilon)$. So for $t_{0}(\varepsilon) \leq t \leq t_{1}(\varepsilon), \quad 0<\varepsilon<\varepsilon_{2}=$ $\min \left(\varepsilon_{D}, d /(2 a), 1,1 / a^{2}\right), 1 \leq \nu \leq l$,

$$
\begin{aligned}
\left|W_{\nu}(t)\right| \leq & K_{E} N_{1} \sum_{i, k=1}^{l} \varepsilon^{\max \left(n_{l}-n_{k}, 0\right)} e^{-\varepsilon^{n_{i} \lambda_{l}\left(t-t_{0}(\varepsilon)\right)}} \\
& +N_{4} \sum_{j=1}^{n_{\nu}} \varepsilon^{j-1}+K_{E} \sum_{i, k=1}^{l} \varepsilon^{\max \left(n_{t}-n_{k}, 0\right)} \frac{\varepsilon^{n_{k}-n_{l}}}{\lambda_{l}} \\
& \times\left\{N_{4}^{2} \sum_{\gamma=1}^{l} \sum_{j=1}^{n_{\gamma}} \varepsilon^{j-1}+2 N_{3}\right\}+\frac{2 K_{E} N_{3}}{\delta} \sum_{i, k=1}^{l} \varepsilon^{\max \left(n_{l}-n_{k}, 0\right)}, \\
\left|W_{\nu}(t)\right| \leq & K_{E} N_{1} l^{2} \\
& +N_{4} n+K_{E} l\left(N_{4}^{2} l n+2 N_{3}\right) \sum_{i=1}^{l} \frac{1}{\lambda_{i}}+\left(\frac{2}{\delta}\right) K_{E} N_{3} l^{2} \\
\equiv & K_{W}
\end{aligned}
$$

where $K_{W}$ is independent of $\varepsilon, a, t_{0}(\varepsilon), t_{1}(\varepsilon)$, and $\nu$. Let

$$
K^{*}=\left\{2 K_{W}+2 N_{1}\left(1+\frac{K_{A}}{\delta}\right)\right\} l \text {. }
$$

Then choosing $a=K^{*}$ and $\varepsilon^{*}=\min \left(\varepsilon_{D}, d /\left(2 K^{*}\right), 1,1 /\left(K^{*}\right)^{2}\right)$, and choosing $t_{1}(\varepsilon)$ such that $(w(t, \varepsilon), z(t, \varepsilon))$ exists on $t_{0}(\varepsilon) \leq t \leq t_{1}(\varepsilon), 0<\varepsilon$ $\leq \varepsilon^{*}$, and such that

$$
\sup _{t_{0}(\varepsilon) \leq t \leq t_{1}(\varepsilon)}\left\{\left|W_{1}(t)\right|,\left|W_{2}(t)\right|, \ldots,\left|W_{l}(t)\right|,|Z(t)|\right\} \leq a \quad\left(=K^{*}\right)
$$

for $0<\varepsilon \leq \varepsilon^{*}$, it then follows that

$$
\sup _{t_{0}(\varepsilon) \leq t \leq t_{1}(\varepsilon)}\left\{\left|W_{1}(t)\right|,\left|W_{2}(t)\right|, \ldots,\left|W_{l}(t)\right|,|Z(t)|\right\} \leq \frac{a}{2 l} \quad\left(=\frac{K^{*}}{2 l}\right)
$$

for $0<\varepsilon \leq \varepsilon^{*}$. Hence, the $t_{1}(\varepsilon)$ picked may be chosen infinite. Consequently, for $0<\varepsilon \leq \varepsilon^{*}$ and for all $\left(b_{01}, b_{02}, \ldots, b_{0 l^{*}}, \xi_{0}\right)$ in $\left(\dot{\circ}_{+} \times R^{I_{0}}\right) \times \stackrel{\circ}{S}_{z}$, the solution $(w(t, \varepsilon), z(t, \varepsilon))$ exists on $t_{0}(\xi) \leq t<\infty$ and

$$
\sup _{t_{0}(\varepsilon) \leq t<\infty}\left\{\left|W_{+}(t)\right|+|Z(t)|\right\} \leq K^{*}
$$

where $W_{+}(t) \equiv\left(W_{1}(t), W_{2}(t), \ldots, W_{l}(t)\right)$.

The rest of the proof of Case $B(M<\infty)$. Now $n_{1} \leq n_{2} \leq \cdots \leq n_{c-1}$ $<n_{c}=n_{c+1}=\cdots=n_{l}(=n)$ from Hypothesis H5. Moreover, positive 
constants $K_{E}(M), \lambda_{l}(M)$ (for $\left.1 \leq i \leq c-1\right)$, and $N_{l}(M)$ exist such that

$$
\begin{aligned}
\left\|U_{\nu k}(t, \tau)\right\| \leq & K_{E}(M) \sum_{i=1}^{c-1} \varepsilon^{\max \left(n_{t}-n_{k}, 0\right)} e^{-\varepsilon^{n_{i}} \lambda_{t}(M)(t-\tau)} \\
& +K_{E}(M) \varepsilon^{n-n_{k}} e^{\varepsilon^{n} N_{t}(M)(t-\tau)}
\end{aligned}
$$

for all $t_{0}(\varepsilon) \leq \tau \leq t_{0}(\varepsilon)+\left(M / \varepsilon^{n}\right), 0<\varepsilon \leq \varepsilon_{D}$, independent of $t_{0}(\varepsilon) \geq 0$ and the chosen $\rho_{+}(t, \varepsilon)$. So for $t_{0}(\varepsilon) \leq t \leq t_{1}(\varepsilon)\left(\leq t_{0}(\varepsilon)+\left(M / \varepsilon^{n}\right)\right)$, $0<\varepsilon \leq \varepsilon_{2}=\min \left(\varepsilon_{D}, d /(2 a), 1,1 / a^{2}\right)$, and $1 \leq \nu \leq l$, we have from (22):

$$
\begin{aligned}
& \left|W_{\nu}(t)\right| \leq K_{E}(M) \sum_{k=1}^{l} \sum_{l=1}^{c-1} \varepsilon^{\max \left(n_{t}-n_{k}, 0\right)} e^{-\varepsilon^{n_{t}} \lambda_{l}(M)\left(t-t_{0}(\varepsilon)\right)} N_{1} \\
& +K_{E}(M) \sum_{k=1}^{l} \varepsilon^{n-n_{k}} e^{\varepsilon^{n} N_{l}\left(t-t_{0}(\varepsilon)\right)} N_{1}+N_{4} \sum_{J=1}^{n_{v}} \varepsilon^{J-1} \\
& +K_{E}(M) \sum_{k=1}^{l} \sum_{i=1}^{c-1} \varepsilon^{\max \left(n_{i}-n_{k}, 0\right)} \frac{\varepsilon^{n_{k}-n_{i}}}{\lambda_{i}(M)} \\
& \times\left\{N_{4}^{2} \sum_{\gamma=1}^{l} \sum_{j=1}^{n_{\gamma}} \varepsilon^{j-1}+2 N_{3}\right\} \\
& +\frac{2 K_{E}(M) N_{3}}{\delta} \sum_{k=1}^{l} \sum_{i=1}^{c-1} \varepsilon^{\max \left(n_{i}-n_{k}, 0\right)} \\
& +\frac{K_{E}(M) l}{N_{l}(M)}\left\{N_{4}^{2} \sum_{\gamma=1}^{l} \sum_{j=1}^{n_{\gamma}} \varepsilon^{j-1}+2 N_{3}\right\} e^{\varepsilon^{n} N_{l}(M)\left(t-t_{0}(\varepsilon)\right)} \\
& +K_{E}(M) \frac{e^{\varepsilon^{n} N_{l}(M)\left(t-t_{0}(\varepsilon)\right)}}{\delta / 2+\varepsilon^{n} N_{l}(M)} N_{3} \sum_{k=1}^{l} \varepsilon^{n-n_{k}} \\
& \left|W_{\nu}(t)\right| \leq K_{E}(M) N_{1} l\left(c-1+e^{M N_{l}(M)}\right)+N_{4} \sum_{j=1}^{n_{\nu}} \varepsilon^{j-1} \\
& +K_{E}(M) l\left(N_{4}^{2} \ln +2 N_{3}\right) \sum_{l=1}^{c-1} \frac{1}{\lambda_{l}(M)} \\
& +\left(\frac{2}{\delta}\right) K_{E}(M) N_{3} l(c-1) \\
& +\frac{K_{E}(M) l}{N_{l}(M)}\left(N_{4}^{2} \ln +2 N_{3}\right) e^{M N_{l}(M)} \\
& +\left(\frac{2}{\delta}\right) K_{E}(M) N_{3} l e^{M N_{l}} \\
& \equiv K_{W}(M, n)
\end{aligned}
$$


where $K_{W}(M, n)$ is independent of $\varepsilon, a, t_{0}(\varepsilon), t_{1}(\varepsilon)$, and $\nu$. Let

$$
K_{n}(M)=2 K_{W}(M, n) l+2 N_{1}\left(1+\left(K_{A} / \delta\right)\right) l .
$$

Then choosing $a=K_{n}(M)$ and

$$
\varepsilon_{n}(M)=\min \left(\varepsilon_{D}, d /\left(2 K_{n}(M)\right), 1,\left(1 /\left\{K_{n}(M)\right\}^{2}\right)\right)
$$

and choosing $t_{1}(\varepsilon)\left(\leq t_{0}(\varepsilon)+\left(M / \varepsilon^{n}\right)\right)$ such that $(w(t, \varepsilon), z(t, \varepsilon))$ exists on $t_{0}(\varepsilon) \leq t \leq t_{1}(\varepsilon), 0<\varepsilon \leq \varepsilon_{n}(M)$, and such that

$$
\sup _{t_{0}(\varepsilon) \leq t \leq t_{1}(\varepsilon)}\left\{\left|W_{1}(t)\right|,\left|W_{2}(t)\right|, \ldots,\left|W_{l}(t)\right|,|Z(t)|\right\} \leq a \quad\left(=K_{n}(M)\right),
$$

it then follows that

$$
\sup _{t_{0}(\varepsilon) \leq t \leq t_{1}(\varepsilon)}\left\{\left|W_{1}(t)\right|,\left|W_{2}(t)\right|, \ldots,\left|W_{l}(t)\right|,|Z(t)|\right\} \leq \frac{a}{2 l} \quad\left(=\frac{K_{n}(M)}{2 l}\right)
$$

for $0<\varepsilon \leq \varepsilon_{n}(M)$. Hence, the $t_{1}(\varepsilon)$ chosen may be picked equal to $t_{0}(\varepsilon)+\left(M / \varepsilon^{n}\right)$. Consequently, for $0<\varepsilon \leq \varepsilon_{n}(M)$ and for all $\left(b_{01}, b_{02}, \ldots, b_{0 l^{*}}, \xi_{0}\right)$ in $\left(\dot{S}_{+} \times R^{I_{0}}\right) \times \stackrel{\circ}{S}_{z}$, the solution $(w(t, \varepsilon), z(t, \varepsilon))$ exists on $t_{0}(\varepsilon) \leq t \leq t_{0}(\varepsilon)+\left(M / \varepsilon^{n}\right)$ and

$$
\sup _{t_{0}(\varepsilon) \leq t \leq t_{0}(\varepsilon)+M / \varepsilon^{n}}\left\{\left|W_{+}(t)\right|+|Z(t)|\right\} \leq K_{n}(M) .
$$

This completes the proof of the theorem.

\section{REFERENCES}

[1] M. Balachandra and P. Sethna, A generalization of the method of averaging for systems with two time scales, Arch. Rational Mech. Anal., 58 (1975), 261-283.

[2] N. Bogoliubov and Yu. Mitropolskii, Asymptotic Methods in the Theory of Non-Linear Oscillations, Gordon and Breach, New York, 1961.

[3] J. Carr, Applications of Centre Manifold Theory, Applied Mathematical Sciences, vol. 35, Springer-Verlag, New York, 1981.

[4] D. Gilsinn, The methods of averaging and domains of stability for integral manifolds, SIAM J. Appl. Math., 29 (1975), 628-660.

[5] K. Landman and S. Rosenblatt, Bifurcation from a multiple eigenvalue and stability of solutions, SIAM J. Appl. Math., 34 (1978), 743-759.

[6] W. Langford, Periodic and steady-state mode interactions lead to tori, SIAM J. Appl. Math., 37 (1979), 22-48.

[7] J. Marsden and M. McCracken, The Hopf Bifurcation and Its Applications, Applied Mathematical Sciences, vol. 19, Springer-Verlag, New York, 1976.

[8] S. Persek and F. Hoppensteadt, Iterated averaging methods for systems of ordinary differential equations with a small parameter, Comm. Pure \& Appl. Math., 31 (1978), 133-156.

[9] S. Persek, Hierarchies of iterated averages for systems of ordinary differential equations with a small parameter, SIAM J. Math. Analysis, 12 (1981), 413-420. 
[10] Asymptotic stability on slow time scales for periodic systems, SIAM J. Appl. Math., 41 (1981), 16-28.

[11] Conditional stability for periodic solutions of periodic systems containing a small parameter, J. Differential Equations, 41 (1981), 163-185.

[12] _ Stability methods for non-periodic solutions of periodic systems, J. Nonlinear Anal., to appear.

[13] A. Poore, On the dynamical behavior of the two-temperature feedback nuclear reactor model, SIAM J. Appl. Math., 30 (1976), 675-686.

[14] N. Rouche, P. Habets, and M. Laloy, Stability Theory by Liapunov's Direct Method, Applied Mathematical Sciences, vol. 22, Springer-Verlag, New York, 1977.

[15] J. Sanders, Asymptotic approximations and extension of time-scales, SIAM J. Math. Analysis, 11 (1980), 758-770.

[16] T. Yoshizawa, Stability Theory and the Existence of Periodic Solutions and Almost Periodic Solutions, Applied Mathematical Sciences, vol. 14, Springer-Verlag, New York, 1975.

Received January 11, 1982.

160 BANBURY ROAD

MineOLA, NY 11501

AND

CBA, ST. JOHN's UNIVERSITY

JAMAICA, NY 11439 


\section{PACIFIC JOURNAL OF MATHEMATICS \\ EDITORS}

DONALD BABBITT (Managing Editor)

University of California.

Los Angeles, CA 90024

Hugo Rossi

University of Utah

Salt Lake City, UT 84112

C. C. Moore and Arthur Ogus

University of California

Berkeley, CA 94720
J. DugundjI

Department of Mathematics

University of Southern California

Los Angeles, CA 90089-1113

R. FinN and H. Samelson

Stanford University

Stanford, CA 94305

\section{ASSOCIATE EDITORS}
R. ARENS
E. F. BECKENBACH
B. H. NeumanN
F. WOLF
K. YoshidA (1906-1982)

\section{SUPPORTING INSTITUTIONS}

UNIVERSITY OF ARIZONA

UNIVERSITY OF BRITISH COLUMBIA

CALIFORNIA INSTITUTE OF TECHNOLOGY

UNIVERSITY OF CALIFORNIA

MONTANA STATE UNIVERSITY

UNIVERSITY OF NEVADA. RENO

NEW MEXICO STATE UNIVERSITY

OREGON STATE UNIVERSITY
UNIVERSITY OF OREGON

UNIVERSITY OF SOUTHERN CALIFORNIA

STANFORD UNIVERSITY

UNIVERSITY OF HAWAII

UNIVERSITY OF TOKYO

UNIVERSITY OF UTAH

WASHINGTON STATE UNIVERSITY

UNIVERSITY OF WASHINGTON 


\section{Pacific Journal of Mathematics}

Vol. 112, No. $1 \quad$ January, 1984

Richard Blaine Barrar and Henry Loeb, Characterizing the divided difference weights for extended complete Tchebycheff systems $\ldots \ldots \ldots \ldots 1$

Harold Bennett and David John Lutzer, Generalized ordered spaces with

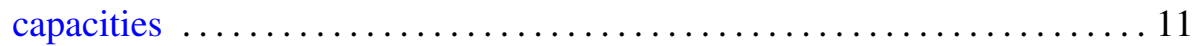

Geoffrey J. Butler and Lynn Harry Erbe, Comparison theorems for second-order operator-valued linear differential equations

Bohumil Cenkl and Richard D. Porter, de Rham theorem with cubical

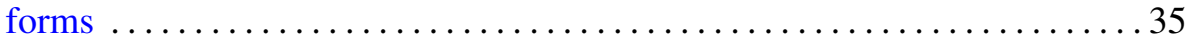

Zvonko Cerin, Characterizing global properties in inverse limits . ....... 49

Thomas Eugene Duchamp and Morris Kalka, Holomorphic foliations and deformations of the Hopf foliation .........................69 69

John Paul Hempel, Homology of coverings $\ldots \ldots \ldots \ldots \ldots \ldots \ldots \ldots \ldots$

Gerald Norman Hile and R. Z. Yeh, Inequalities for eigenvalues of the

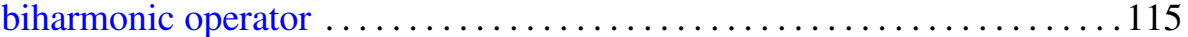

Kenneth Irwin Joy, A description of the topology on the dual space of a

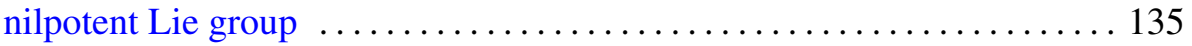

Alex Kumjian, On localizations and simple $C^{*}$-algebras $\ldots \ldots \ldots \ldots \ldots 141$

Bernardus de Pagter, The space of extended orthomorphisms in a Riesz

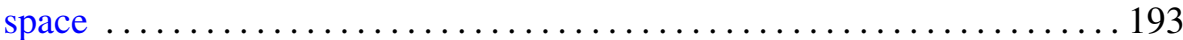

Stephen C. Persek, Iterated averaging for periodic systems with hidden

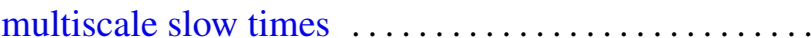

David Francis Rearick, Divisibility of arithmetic functions . . . . . . . . . 237

Masaaki Suzuki, The intrinsic metrics on the circular domains in $\mathbf{C}^{n}$ 